\title{
Nodal Solutions of the Brezis-Nirenberg Problem in Dimension 6
}

\author{
Angela Pistoia $^{1, *}$ and Giusi Vaira ${ }^{2}$ \\ ${ }^{1}$ Dipartimento di Scienze di Base e Applicate per l'Ingegneria, Sapienza Università \\ di Roma, Via Scarpa 16, 00161 Roma, Italy \\ 2 Dipartimento di Matematica, Università degli studi di Bari "Aldo Moro", via Edoardo \\ Orabona 4,70125 Bari, Italy
}

Received 19 October 2020; Accepted (in revised version) 4 January 2021

Abstract. We show that the classical Brezis-Nirenberg problem

$$
\begin{array}{ll}
-\Delta u=u|u|+\lambda u & \text { in } \Omega, \\
u=0 & \text { on } \partial \Omega,
\end{array}
$$

when $\Omega$ is a bounded domain in $\mathbb{R}^{6}$ has a sign-changing solution which blows-up at a point in $\Omega$ as $\lambda$ approaches a suitable value $\lambda_{0}>0$.

Key Words: Sign-changing solutions, blow-up phenomenon, Ljapunov-Schmidt reduction, Transversality theorem.

AMS Subject Classifications: 35B44, 58C15

\section{Introduction}

Brezis and Nirenberg in their famous paper [6] introduced the problem

$$
\begin{array}{ll}
-\Delta u=|u|^{\frac{4}{n-2}} u+\lambda u & \text { in } \Omega, \\
u=0 & \text { on } \partial \Omega,
\end{array}
$$

where $\Omega$ is a smooth bounded domain in $\mathbb{R}^{n}$ and $n \geq 3$. A huge number of results concerning (1.1) has been obtained since then. Let us summarize the most relevant results which are also connected with the topic of the present paper.

First of all, the classical Pohozaev's identity ensures that (1.1) does not have any solutions if $\lambda \leq 0$ and $\Omega$ is a star-shaped domain. A simple argument shows that problem

*Corresponding author. Email addresses: angela.pistoia@uniroma1 . it (A. Pistoia), giusi .vaira@uniba.it (G. Vaira) 
(1.1) does not have any positive solutions if $\lambda \geq \lambda_{1}(\Omega)$, where $\lambda_{1}(\Omega)$ is the first eigenvalue of $-\Delta$ with Dirichlet boundary condition. The existence of a least energy positive solution $u_{\lambda}$ to (1.1), i.e., a solution which achieves the infimum

$$
m_{\lambda}:=\inf _{u \in H_{0}^{1}(\Omega) \backslash\{0\}} \frac{\int_{\Omega_{\theta}}\left(|\nabla u|^{2}-\lambda u^{2}\right) d x}{\left(\int_{\Omega}|u|^{p+1} d x\right)^{\frac{2}{p+1}}}
$$

has been proved by Brezis and Nirenberg in [6] when $\lambda \in\left(0, \lambda_{1}(\Omega)\right)$ in dimension $n \geq 4$ and when $\lambda \in\left(\lambda^{*}(\Omega), \lambda_{1}(\Omega)\right)$ in dimension $n=3$, where $\lambda^{*}(\Omega)>0$ depends on the domain $\Omega$. If $\Omega$ is the ball then $\lambda^{*}(\Omega)=\frac{1}{4} \lambda_{1}(\Omega)$ (see [6]), while the general case has been treated by Druet in [12]. The existence of a sign-changing solution has been proved by Cerami, Solimini and Struwe in [9] when $\lambda \in\left(0, \lambda_{1}(\Omega)\right)$ and $n \geq 6$ and by Capozzi, Fortunato and Palmieri in [8] when $\lambda \geq \lambda_{1}(\Omega)$ and $n \geq 4$.

There is a wide literature about the study of the asymptotic profile of the solutions when the parameter $\lambda$ approaches either zero or some strictly positive values depending on the dimension $n$ and the domain $\Omega$. In the following, we will focus on the existence of solutions which exhibit a positive or negative blow-up phenomenon as $\lambda$ approaches some particular values.

When the parameter $\lambda$ approaches zero, positive and sign-changing solutions which blow-up positively or negatively at one or more points in $\Omega$ do exist provided the dimension $n \geq 4$. Rey in [24], Musso and Pistoia in [19] and Esposito, Pistoia and Vétois in [13] built solutions to (1.1) with simple positive or negative blow-up points, i.e., around each point the solution looks like a positive or a negative standard bubble. Here the standard bubbles are the functions

$$
\begin{aligned}
& U_{\delta, \xi}(x):=\alpha_{n} \frac{\delta^{\frac{n-2}{2}}}{\left(\delta^{2}+|x-\xi|^{2}\right)^{\frac{n-2}{2}}} \text { with } \delta>0, \quad \xi \in \mathbb{R}^{n}, \\
& \alpha_{n}:=(n(n-2))^{\frac{n-2}{4}},
\end{aligned}
$$

which are the only positive solutions of the equation

$$
-\Delta U=U^{\frac{n+2}{n-2}}
$$

in $\mathbb{R}^{n}$ (see $[4,7,28]$ ) More precisely, if $\lambda$ is small enough problem (1.1) has a positive solution which blows-up at one point (see [24] if $n \geq 5$ and [13] if $n=4$ ) and a sign-changing solution which blows-up positively and negatively at two different points (see [19] if $n \geq 5$ ). As far as we know the existence of multiple concentration in the case $n=4$ is still open. If $n=3$ positive solutions of (1.1) blowing-up at a single point when the parameter $\lambda$ approaches a strictly positive number have been found by Del Pino, Dolbeault and Musso in [11]. Moreover, sign-changing solutions having both positive and negative blow-up points can be constructed arguing as Musso and Salazar in [20], where they found solutions which blow-up at more points when $\lambda$ is close to a suitable strictly positive number. In higher dimension $n \geq 7$ Premoselli [22] found an arbitrary large number 
of sign-changing solutions to (1.1) with a towering blow-up point in $\Omega$, i.e., around the point the solution likes look like the superposition of bubbles of alternating sign (see also Iacopetti and Vaira [16]). In particular, if $\Omega$ is a ball these solutions are nothing but the radially symmetric nodal solutions. Conversely, if $\Omega$ is the ball in low dimension $n=3,4,5,6$, Atkinson, Brezis and Peletier in [3] proved that problem (1.1) does not have any sign-changing radial solutions when $\lambda \in\left(0, \lambda_{*}\right)$ where $\lambda_{*}$ depends on the dimension $n$ (see also Iacopetti and Pacella [15] and Dammak [10]). In particular, we expect that in low dimension the blowing-up phenomenon takes places when $\lambda$ approaches a positive value different from zero. In fact Iacopetti and Vaira in [17] proved that if $n=4,5$ and $\lambda$ approaches the first eigenvalue $\lambda_{1}(\Omega)$ the problem (1.1) has a sign-changing solution which blows-up at the origin and shares the shape of the positive first eigenfunction associated with $\lambda_{1}(\Omega)$ far away. So a natural question arises: is it possible to find a signchanging blowing-up solution of (1.1) in dimension $n=6$ when $\lambda$ approaches some strictly positive number?

In the present paper, we give a positive answer. In order to state our result, we need to introduce some notation and the assumptions.

Let $u_{0}$ be a solution to

$$
\begin{cases}-\Delta u_{0}=\left|u_{0}\right| u_{0}+\lambda_{0} u_{0} & \text { in } \Omega, \\ u_{0}=0 & \text { on } \partial \Omega .\end{cases}
$$

If $\xi_{0} \in \Omega$ is such that $\max _{\Omega} u_{0}=u_{0}\left(\xi_{0}\right)>0$, we suppose that

$$
\lambda_{0}=2 u_{0}\left(\xi_{0}\right)
$$

We assume that $u_{0}$ is non-degenerate, i.e.,

$$
\left\{\begin{array}{ll}
-\Delta v=\left(2\left|u_{0}\right|+\lambda_{0}\right) v & \text { in } \Omega \\
v=0 & \text { on } \partial \Omega
\end{array} \Rightarrow v \equiv 0 .\right.
$$

If $v_{0}$ solves

$$
\begin{cases}-\Delta v_{0}-\left(2\left|u_{0}\right|+\lambda_{0}\right) v_{0}=u_{0} & \text { in } \Omega, \\ v_{0}=0 & \text { on } \partial \Omega,\end{cases}
$$

we require that

$$
2 v_{0}\left(\xi_{0}\right) \neq 1
$$

We will show that the problem

$$
\begin{cases}-\Delta u=u|u|+\left(\lambda_{0}+\epsilon\right) u & \text { in } \Omega, \\ u=0 & \text { on } \partial \Omega\end{cases}
$$


where $\Omega$ is a bounded domain in $\mathbb{R}^{6}$ has a sign-changing solution which blows-up at $\xi_{0}$ as $|\epsilon|$ approaches zero (note that $\epsilon$ is not necessarily positive) and shares the shape of $u_{0}$ far away from $\xi_{0}$. More precisely our existence result reads as follows.

Theorem 1.1. Assume (1.4), (1.5) and (1.7). There exists $\varepsilon_{0}>0$ such that

1. if $1-2 v_{0}\left(\xi_{0}\right)>0$ and $\epsilon \in\left(0, \varepsilon_{0}\right)$,

2. if $1-2 v_{0}\left(\xi_{0}\right)<0$ and $\epsilon \in\left(-\varepsilon_{0}, 0\right)$,

then there exists a sign-changing solution $u_{\epsilon}$ of the problem (1.8), which blows-up at the point $\xi_{0}$ as $\epsilon \rightarrow 0$. More precisely

$$
u_{\epsilon}(x)=u_{0}(x)+\epsilon v_{0}(x)-P U_{\delta_{\epsilon}, \xi_{\epsilon}}(x)+\phi_{\epsilon}(x)
$$

with as $\epsilon \rightarrow 0$

$$
\delta_{\epsilon}|\epsilon|^{-1} \rightarrow d>0, \quad \xi_{\epsilon} \rightarrow \xi_{0} \quad \text { and } \quad\left\|\phi_{\epsilon}\right\|_{H_{0}^{1}(\Omega)}=\mathcal{O}\left(\left.\epsilon^{2}|\ln | \epsilon\right|^{\frac{2}{3}}\right) .
$$

Here $P U_{\delta, \xi}$ denotes the projection onto $H_{0}^{1}(\Omega)$ of the standard bubble $U_{\delta, \xi}$ defined in (1.2), i.e., $-\Delta P U_{\delta, \xi}=U_{\delta, \xi}^{2}$ in $\Omega$ with $P U_{\delta, \xi}=0$ on $\partial \Omega$.

It is natural to ask for which domains $\Omega$ the assumptions (1.4), (1.5) and (1.7) hold true. If $\Omega$ is the ball and $u_{0}$ is the positive solution they are all satisfied (see [27] for (1.5), [1] for (1.4) and (1.7)). More in general, we can only prove that assumptions (1.4) and (1.5) are satisfied for most domains $\Omega$ (see Theorem 1.2) when $u_{0}$ is the least energy positive solution to (1.3). It would be interesting to prove that (1.7) also holds for generic domains.

Let us state our generic result. Let $\Omega_{0}$ be a bounded and smooth domain in $\mathbb{R}^{6}$ and let $D$ be an open neighbourhood of $\overline{\Omega_{0}}$. Set $\Omega_{\theta}:=\Theta\left(\Omega_{0}\right)$ where $\Theta=I+\theta, \theta \in C^{3, \alpha}\left(\bar{D}, \mathbb{R}^{6}\right)$ with $\|\theta\|_{2, \alpha} \leq \rho$, with $\alpha \in(0,1)$ and $\rho$ small enough. Let us consider the problem on the perturbed domain $\Omega_{\theta}$

$$
\begin{array}{ll}
\Delta u+\lambda u+|u| u=0 & \text { in } \Omega_{\theta}, \\
u=0 & \text { on } \partial \Omega_{\theta} .
\end{array}
$$

Theorem 1.2. The set

$$
\begin{gathered}
\Xi:=\left\{\theta \in C^{3, \alpha}\left(\bar{D}, \mathbb{R}^{6}\right): \text { if } \lambda>0 \text { and } u \in H_{0}^{1}\left(\Omega_{\theta}\right) \text { solves }(1.9)\right. \\
\text { then } u \text { is non-degenerate }\}
\end{gathered}
$$

is a residual subset in $C^{3, \alpha}\left(\bar{D}, \mathbb{R}^{6}\right)$, i.e., $C^{3, \alpha}\left(\bar{D}, \mathbb{R}^{6}\right) \backslash \Xi$ is a countable union of close subsets without interior points.

Moreover, if $\lambda \in\left(0, \lambda_{1}\left(\Omega_{\theta}\right)\right)$ and $u_{\lambda}$ denotes the least energy positive solution of (1.9), for any $\theta \in \Xi$ there exists $\lambda_{\theta} \in\left(0, \lambda_{1}\left(\Omega_{\theta}\right)\right)$ such that

$$
\lambda_{\theta}=2 \max _{\Omega_{\theta}} u_{\lambda_{\theta}}
$$


The proof of Theorem 1.1 is based upon the well-known Ljapunov-Schmidt reduction. In Section 2 we describe the main steps of the proof by omitting many details which can be found up to minor changes in the quoted papers. We only prove what cannot be immediately deduced by known results. In particular, we point out the careful construction of the ansatz (2.3) which has to be refined up to a second order and the delicate estimate of the reduced energy (2.7) given in Proposition 2.2 whose leading term (2.8) arises from the interaction between the bubble and the second order term in the ansatz.

The proof of Theorem 1.2 relies on a classical transversality argument and it is carried out in Section 3.

\section{The existence of a sign-changing solution}

\subsection{Setting of the problem and the choice of the ansatz}

In what follows we denote by

$$
(u, v):=\int_{\Omega} \nabla u \nabla v d x, \quad\|u\|:=\left(\int_{\Omega}|\nabla u|^{2} d x\right)^{\frac{1}{2}} \quad \text { and } \quad|u|_{r}:=\left(\int_{\Omega}|u|^{r} d x\right)^{\frac{1}{r}},
$$

the inner product and its correspond norm in $H_{0}^{1}(\Omega)$ and the standard norm in $L^{r}(\Omega)$, respectively. When $A \neq \Omega$ is any Lebesgue measurable set we specify the domain of integration by using $\|u\|_{A},|u|_{r, A}$.

Let $(-\Delta)^{-1}: L^{\frac{3}{2}}(\Omega) \rightarrow H_{0}^{1}(\Omega)$ be the operator defined as the unique solution of the equation

$$
\begin{array}{ll}
-\Delta u=v & \text { in } \Omega, \\
u=0 & \text { on } \partial \Omega .
\end{array}
$$

By the Holder inequality it follows that

$$
\left\|(-\Delta)^{-1}(v)\right\| \leq C|v|_{\frac{3}{2}}, \quad \forall v \in L^{\frac{3}{2}}(\Omega),
$$

for some positive constant $C$, which does not depend on $v$. Hence we can rewrite problem (1.8) as

$$
u=(-\Delta)^{-1}\left[f(u)+\left(\lambda_{0}+\epsilon\right) u\right], \quad u \in H_{0}^{1}(\Omega),
$$

with $f(u)=|u| u$.

Next we remind the expansion of the projection of the bubble. We denote by $G(x, y)$ the Green's function of the Laplace operator given by

$$
G(x, y)=\frac{1}{4 \omega_{6}}\left(\frac{1}{|x-y|^{4}}-H(x, y)\right)
$$


where $\omega_{6}$ denotes the surface area of the unit sphere in $\mathbb{R}^{6}$ and $H$ is the regular part of the Green's function, namely for all $y \in \Omega, H(x, y)$ satisfies

$$
\begin{array}{ll}
\Delta H(x, y)=0 & \text { in } \Omega, \\
H(x, y)=\frac{1}{|x-y|^{4}}, & x \in \partial \Omega .
\end{array}
$$

It is known that the following expansion holds (see [24])

$$
P U_{\delta, \xi}(x)=U_{\delta, \xi}(x)-\alpha_{6} \delta^{2} H(x, \xi)+\mathcal{O}\left(\delta^{4}\right) \quad \text { as } \delta \rightarrow 0
$$

uniformly with respect to $\xi$ in compact sets of $\Omega$.

Moreover we recall (see [5]) that every solution to the linear equation

$$
-\Delta \psi=2 U_{\delta, \xi} \psi \quad \text { in } \mathbb{R}^{6}
$$

is a linear combination of the functions $Z_{\delta, \xi^{\prime}}^{j} j=0, \cdots, 6$ given by

$$
\begin{aligned}
& Z_{\delta, \xi}^{0}(x)=\partial_{\delta} U_{\delta, \xi}(x)=2 \alpha_{6} \delta \frac{|x-\xi|^{2}-\delta^{2}}{\left(\delta^{2}+|x-\xi|^{2}\right)^{3}} \\
& Z_{\delta, \xi}^{j}(x)=\partial_{\xi_{j}} U_{\delta, \xi}(x)=4 \alpha_{6} \delta^{2} \frac{x_{j}-\xi_{j}}{\left(\delta^{2}+|x-\xi|^{2}\right)^{3}}, \quad j=1, \cdots, 6 .
\end{aligned}
$$

If we denote by $P Z_{\delta, \xi}^{j}$ the projection of $Z_{\delta, \xi}^{j}$ onto $H_{0}^{1}(\Omega)$, i.e.,

$$
\begin{array}{ll}
-\Delta P Z_{\delta, \xi}^{j}=2 U_{\delta, \xi} Z_{\delta, \xi}^{j} & \text { in } \Omega, \\
P Z_{\delta, \xi}^{j}=0 & \text { on } \partial \Omega,
\end{array}
$$

elliptic estimates give

$$
\begin{array}{ll}
P Z_{\delta, \xi}^{0}(x)=Z_{\delta, \xi}^{0}-2 \delta \alpha_{6} H(x, \xi)+\mathcal{O}\left(\delta^{3}\right) & \text { as } \delta \rightarrow 0, \\
P Z_{\delta, \xi}^{j}(x)=Z_{\delta, \xi}^{j}-\delta^{2} \alpha_{6} \partial_{\xi_{j}} H(x, \xi)+\mathcal{O}\left(\delta^{4}\right), & j=1, \cdots, 6 \text { as } \delta \rightarrow 0,
\end{array}
$$

uniformly with respect to $\xi$ in compact sets of $\Omega$.

We look for a solution of (1.8) of the form

$$
u_{\epsilon}(x)=\underbrace{u_{0}(x)+\epsilon v_{0}-P U_{\delta, \xi}(x)}_{:=W_{\delta, \tilde{\xi}}}+\phi_{\epsilon}(x),
$$

where $\delta, \xi$ are chosen so that

$$
\delta=|\epsilon| d \quad \text { with } d \in\left(\sigma, \frac{1}{\sigma}\right) \text { and } \xi=\xi_{0}+\sqrt{\delta} \eta \text { with }|\eta| \leq \frac{1}{\sigma}, \text { where } \sigma>0 \text { is small, }
$$


and $\phi_{\epsilon}$ is a remainder term, which is small as $\epsilon \rightarrow 0$ which belongs to the space $\mathcal{K}_{\delta, \xi}^{\frac{1}{2}}$ defined as follows.

Now let us define

$$
\begin{aligned}
\mathcal{K}_{\delta, \xi} & :=\operatorname{span}\left\{P Z_{\delta, \xi}^{j}: j=0, \cdots, 6\right\}, \\
\mathcal{K}_{\delta, \xi}^{\perp} & :=\left\{\phi \in H_{0}^{1}(\Omega):\left(\phi, P Z_{\delta, \xi}^{j}\right)=0, j=0, \cdots, 6\right\} .
\end{aligned}
$$

Let us denote by $\Pi_{\delta, \xi}$ and $\Pi_{\delta, \xi}^{\perp}$ the projection of $H_{0}^{1}(\Omega)$ on $\mathcal{K}_{\delta, \xi}$ and $\mathcal{K}_{\delta, \xi}^{\perp}$ respectively.

Then solving problem (2.1) is equivalent to solve the system

$$
\begin{aligned}
& \Pi_{\delta, \xi}^{\perp}\left\{u_{\epsilon}(x)-(-\Delta)^{-1}\left[f\left(u_{\epsilon}\right)+\lambda u_{\epsilon}\right]\right\}=0, \\
& \Pi_{\delta, \xi}\left\{u_{\epsilon}(x)-(-\Delta)^{-1}\left[f\left(u_{\epsilon}\right)+\lambda u_{\epsilon}\right]\right\}=0 .
\end{aligned}
$$

\subsection{The remainder term: solving Eq. (2.5a)}

Eq. (2.5a) can be written as

$$
\mathcal{L}_{\delta, \xi}\left(\phi_{\epsilon}\right)+\mathcal{R}_{\delta, \xi}+\mathcal{N}_{\delta, \xi}\left(\phi_{\epsilon}\right)=0
$$

where

$$
\mathcal{L}_{\delta, \xi}\left(\phi_{\epsilon}\right)=\Pi_{\delta, \xi}^{\perp}\left\{\phi_{\epsilon}(x)-(-\Delta)^{-1}\left[f^{\prime}\left(W_{\delta, \xi}\right) \phi_{\epsilon}+\lambda \phi_{\epsilon}\right]\right\}
$$

is the linearized operator at the approximate solution,

$$
\mathcal{R}_{\delta, \xi}=\Pi_{\delta, \xi}^{\perp}\left\{W_{\delta, \xi}(x)-(-\Delta)^{-1}\left[f\left(W_{\delta, \xi}\right)+\lambda W_{\delta, \xi}\right]\right\}
$$

is the error term and

$$
\mathcal{N}_{\delta, \xi}\left(\phi_{\epsilon}\right)=\Pi_{\delta, \xi}^{\perp}\left\{-(-\Delta)^{-1}\left[f\left(W_{\delta, \xi}+\phi_{\epsilon}\right)-f\left(W_{\delta, \xi}\right)-f^{\prime}\left(W_{\delta, \xi}\right) \phi_{\epsilon}\right]\right\}
$$

is a quadratic term in $\phi_{\epsilon}$.

First of all, we estimate the size of the error term $\mathcal{R}_{\delta, \xi}$.

Lemma 2.1. For any $\sigma>0$ there exist $c>0$ and $\varepsilon_{0}>0$ such that for any $d>0$ and $\eta \in \mathbb{R}^{6}$ satisfying (2.4) and for any $\epsilon \in\left(-\varepsilon_{0}, \varepsilon_{0}\right)$

$$
\left\|\mathcal{R}_{\delta, \xi}\right\| \leq\left. c \epsilon^{2}|\ln | \epsilon\right|^{\frac{2}{3}} .
$$

Proof. First we remark that

$$
\begin{aligned}
- & \Delta W_{\delta, \xi}-\left|W_{\delta, \xi}\right| W_{\delta, \xi}-\left(\lambda_{0}+\epsilon\right) W_{\delta, \xi} \\
=- & \Delta u_{0}-\epsilon \Delta v_{0}-U_{\delta, \xi}^{2}-\left|u_{0}+\epsilon v_{0}-P U_{\delta, \xi}\right|\left(u_{0}+\epsilon v_{0}-P U_{\delta, \xi}\right) \\
& -\lambda_{0} u_{0}-\lambda_{0} \epsilon v_{0}+\left(\lambda_{0}+\epsilon\right) P U_{\delta, \xi}-\epsilon u_{0}-\epsilon^{2} v_{0} \\
=- & \left|u_{0}+\epsilon v_{0}-P U_{\delta, \xi}\right|\left(u_{0}+\epsilon v_{0}-P U_{\delta, \xi}\right)-U_{\delta, \xi}^{2}+\left|u_{0}\right| u_{0} \\
& +\epsilon \underbrace{\left(-\Delta v_{0}-\lambda_{0} v_{0}-u_{0}\right)}_{=2\left|u_{0}\right| v_{0} \text { because of }(1.6)}+\left(\lambda_{0}+\epsilon\right) P U_{\delta, \xi}-\epsilon^{2} v_{0} .
\end{aligned}
$$


By the continuity of $\Pi_{\delta, \xi}^{\perp}$ we get that

$$
\begin{aligned}
\left\|\mathcal{R}_{\delta, \xi}\right\| \leq & c\left|-\Delta W_{\delta, \xi}-f\left(W_{\delta, \xi}\right)-\lambda W_{\delta, \xi}\right|_{\frac{3}{2}} \\
\leq c & \underbrace{|-| u_{0}+\epsilon v_{0}-P U_{\delta, \xi}\left|\left(u_{0}+\epsilon v_{0}-P U_{\delta, \xi}\right)-P U_{\delta, \xi}^{2}+\right| u_{0}\left|u_{0}+2 \epsilon\right| u_{0}\left|v_{0}\right|_{\frac{3}{2}}}_{(I)} \\
& +\underbrace{c\left|P U_{\delta, \xi}^{2}-U_{\delta, \xi}^{2}\right|_{\frac{3}{2}}}_{(I I)}+\left(\lambda_{0}+\epsilon\right)\left|P U_{\delta, \xi}\right|_{\frac{3}{2}}+\underbrace{\epsilon^{2}\left|v_{0}\right|_{\frac{3}{2}}}_{:=\mathcal{O}\left(\epsilon^{2}\right)} .
\end{aligned}
$$

First of all, we point out that

$$
\left|P U_{\delta, \xi}\right|_{\frac{3}{2}} \leq c\left|U_{\delta, \xi}\right|_{\frac{3}{2}} \leq c \delta^{2}|\ln \delta|^{\frac{2}{3}}
$$

and by (2.2)

$$
(I I) \leq c(\int_{\Omega} \underbrace{\left|P U_{\delta, \xi}-U_{\delta, \xi}\right|^{\frac{3}{2}}}_{=O\left(\delta^{2}\right)} \underbrace{\left|P U_{\delta, \xi}+U_{\delta, \xi}\right|^{\frac{3}{2}}}_{\leq c U_{\delta, \xi}})^{\frac{2}{3}} \leq c \delta^{2}\left(\int_{\Omega}\left|U_{\delta, \xi}\right|^{\frac{3}{2}} d x\right)^{\frac{2}{3}}=\mathcal{O}\left(\delta^{4}|\ln \delta|^{\frac{2}{3}}\right) .
$$

First let us estimate $(I)$ in $B(\xi, \sqrt{\delta})$ and $\Omega \backslash B(\xi, \sqrt{\delta})$ :

$$
\begin{aligned}
&(I) \leq c\left(\int_{B(\xi, \sqrt{\delta})}|| u_{0}+\epsilon v_{0}-P U_{\delta, \xi}\left|\left(u_{0}+\epsilon v_{0}-P U_{\delta, \xi}\right)\right|+\left.\left(P U_{\delta, \xi}\right)^{2}\right|^{\frac{3}{2}}\right)^{\frac{2}{3}} \\
&+c \underbrace{\left(\int_{B(\xi, \sqrt{\delta})}|| u_{0}\left|u_{0}+2 \epsilon\right| u_{0}\left|v_{0}\right|^{\frac{3}{2}} d x\right)^{\frac{2}{3}}}_{=\mathcal{O}\left(\delta^{2}\right)} \\
&+c\left(\int_{\Omega \backslash B(\xi, \sqrt{\delta})}|| u_{0}+\epsilon v_{0}-P U_{\delta, \xi}\left|\left(u_{0}+\epsilon v_{0}-P U_{\delta, \xi}\right)-\right| u_{0}\left|u_{0}-2\right| u_{0} \mid\left(\epsilon v_{0}-P U_{\delta, \xi}\right)^{\frac{3}{2}}\right)^{\frac{2}{3}} \\
&+c\left(\int_{\Omega \backslash B(\xi, \sqrt{\delta})}\left|\left(P U_{\delta, \xi}\right)^{2}+2\right| u_{0}\left|P U_{\delta, \xi}\right|^{\frac{3}{2}} d x\right)^{\frac{2}{3}} \\
&=\mathcal{O}\left(\delta^{2}|\ln \delta|^{\frac{2}{3}}\right),
\end{aligned}
$$

since by mean value Theorem (here $\theta \in[0,1]$ )

$$
\begin{aligned}
& \int_{B(\xi, \sqrt{\delta})}|| u_{0}+\epsilon v_{0}-P U_{\delta, \xi}\left|\left(u_{0}+\epsilon v_{0}-P U_{\delta, \xi}\right)+\left(P U_{\delta, \xi}\right)^{2}\right|^{\frac{3}{2}} \\
= & 2 \int_{B(\xi, \sqrt{\delta})}\left|\left(\theta\left(u_{0}+\epsilon v_{0}\right)-P U_{\delta, \xi}\right)\left(u_{0}+\epsilon v_{0}\right)\right|^{\frac{3}{2}} d x \\
\leq & c \underbrace{\int_{B(\xi, \sqrt{\delta})}\left|P U_{\delta, \xi}\right|^{\frac{3}{2}} d x}_{=\mathcal{O}\left(\delta^{3}|\log \delta|\right)}+c \underbrace{\int_{B(\xi, \sqrt{\delta})}\left|u_{0}+\epsilon v_{0}\right|^{3} d x}_{=\mathcal{O}\left(\delta^{3}\right)}
\end{aligned}
$$


and by the inequality

$$
|| a+b|(a+b)-| a|a-2| a|b| \leq 7 b^{2} \quad \text { for any } a, b \in \mathbb{R},
$$

and

$$
\begin{aligned}
& \int_{\Omega \backslash B(\xi, \sqrt{\delta})}|| u_{0}+\epsilon v_{0}-P U_{\delta, \xi}\left|\left(u_{0}+\epsilon v_{0}-P U_{\delta, \xi}\right)-\right| u_{0}\left|u_{0}-2\right| u_{0}\left|\left(\epsilon v_{0}-P U_{\delta, \xi}\right)\right|^{\frac{3}{2}} \\
& \leq c \int_{\Omega \backslash B(\xi, \sqrt{\delta})}\left|\epsilon v_{0}-P U_{\delta, \xi}\right|^{3} d x \\
& \leq c \underbrace{\int_{\Omega \backslash B(\xi, \sqrt{\delta})}\left|\epsilon v_{0}\right|^{3} d x}_{=\mathcal{O}\left(\epsilon^{3}\right)}+c \underbrace{\int_{\Omega \backslash B(\xi, \sqrt{\delta})}\left|U_{\delta, \xi}\right|^{3} d x}_{=\mathcal{O}\left(\delta^{3}\right)}, \\
& \left(\int_{\Omega \backslash B(\xi, \sqrt{\delta})}|| u_{0}+\epsilon v_{0}-P U_{\delta, \xi}\left|\left(u_{0}+\epsilon v_{0}-P U_{\delta, \xi}\right)-\right| u_{0}\left|u_{0}-2\right| u_{0}\left|\left(\epsilon v_{0}-P U_{\delta, \xi}\right)\right|^{\frac{3}{2}}\right)^{\frac{2}{3}} \\
& =\mathcal{O}\left(\epsilon^{2}\right), \\
& \int_{\Omega \backslash B(\xi, \sqrt{\delta})}|| P U_{\delta, \xi}\left|\left(P U_{\delta, \xi}\right)+2\right| u_{0}\left|P U_{\delta, \xi}\right|^{\frac{3}{2}} \\
& \leq c \underbrace{\int_{\Omega(\xi(\xi, \sqrt{\delta})}\left|U_{\delta, \xi}\right|^{3} d x}_{=\mathcal{O}\left(\delta^{3}\right)}+\underbrace{\int_{\Omega \backslash B(\xi, \sqrt{\delta})}\left|U_{\delta, \xi}\right|^{\frac{3}{2}} d x}_{=\mathcal{O}\left(\delta^{3}|\log \delta|\right)}
\end{aligned}
$$

which ends the proof.

Next we analyze the invertibility of the linear operator $\mathcal{L}_{\delta, \xi}$ (see for example [30], Lemma 2.4 or [25], Lemma 4.2).

Lemma 2.2. For any $\sigma>0$ there exist $c>0$ and $\varepsilon_{0}>0$ such that for any $d>0$ and $\eta \in \mathbb{R}^{6}$ satisfying (2.4) and for any $\epsilon \in\left(-\varepsilon_{0}, \varepsilon_{0}\right)$

$$
\left\|\mathcal{L}_{\delta, \xi}(\phi)\right\| \geq c\|\phi\| \quad \text { for any } \phi \in \mathcal{K}_{\delta, \xi}^{\perp} .
$$

Moreover, $\mathcal{L}_{\delta, \xi}$ is invertible and $\left\|\mathcal{L}_{\delta, \xi}^{-1}\right\| \leq \frac{1}{c}$.

We are in position now to find a solution of Eq. (2.5a) whose proof relies on a standard contraction mapping argument (see for example [19, Proposition 1.8] and [18, Proposition 2.1])

Proposition 2.1. For any $\sigma>0$ there exist $c>0$ and $\varepsilon_{0}>0$ such that for any $d>0$ and $\eta \in \mathbb{R}^{6}$ satisfying (2.4) and for any $\epsilon \in\left(-\varepsilon_{0}, \varepsilon_{0}\right)$, there exists a unique $\phi_{\epsilon}=\phi_{\epsilon}(d, \eta) \in \mathcal{K}_{\delta, \xi}^{\perp}$ solution to (2.5a) which is continuously differentiable with respect to $d$ and $\eta$ and such that

$$
\left\|\phi_{\epsilon}\right\| \leq c \epsilon^{2}|\ln | \epsilon||^{\frac{2}{3}} .
$$




\subsection{The reduced problem: solving Eq. (2.5b)}

To solve Eq. (2.5b), we shall find the parameter $\delta$ and the point $\xi \in \Omega$ as in (2.4), i.e., $d>0$ and $\eta \in \mathbb{R}^{6}$, so that $(2.5 b)$ is satisfied. It is well known that this problem has a variational structure, in the sense that solutions of $(2.5 \mathrm{~b})$ reduces to find critical points to some given explicit finite dimensional functional. Indeed, let $J_{\epsilon}: H_{0}^{1}(\Omega) \rightarrow \mathbb{R}$ defined by

$$
J_{\epsilon}(u):=\frac{1}{2} \int_{\Omega}|\nabla u|^{2} d x-\frac{\lambda}{2} \int_{\Omega} u^{2} d x-\frac{1}{3} \int_{\Omega}|u|^{3} d x
$$

and let $\tilde{J}_{\epsilon}: \mathbb{R}_{+} \times \mathbb{R}^{6} \rightarrow \mathbb{R}$ be the reduced energy which is defined by

$$
\tilde{J}_{\epsilon}(d, \eta)=J_{\epsilon}\left(W_{\delta, \xi}+\phi_{\epsilon}\right) .
$$

Proposition 2.2. For any $\sigma>0$ there exists $\varepsilon_{0}>0$ such that for any $\epsilon \in\left(-\varepsilon_{0}, \varepsilon_{0}\right)$

$$
\tilde{J}_{\epsilon}(d, \eta)=\mathfrak{c}_{0}(\epsilon)+|\epsilon|^{3} Y(d, \eta)+o\left(|\epsilon|^{3}\right)
$$

with

$$
\mathrm{Y}(d, \eta):=\operatorname{sgn}(\epsilon)\left(1-2 v_{0}\left(\xi_{0}\right)\right) d^{2} \mathfrak{a}_{1}+d^{3}\left(\mathfrak{a}_{2}\left\langle D^{2} u_{0}\left(\xi_{0}\right) \eta, \eta\right\rangle-\mathfrak{a}_{3}\right),
$$

uniformly with respect to $(d, \eta)$ which satisfies (2.4), where the $\mathfrak{c}_{0}(\epsilon)$ depends only on $\epsilon$ and the $\mathfrak{a}_{i}$ 's are positive constants. Moreover, if $(d, \eta)$ is a critical point of $\tilde{J}_{\epsilon}$, then $W_{\delta, \xi}+\phi_{\epsilon}$ is a solution of (1.8).

Proof. It is quite standard to prove that if $(d, \eta)$ satisfies $(2.4)$ and is a critical point of $\tilde{J}_{\epsilon}$, then $W_{\delta, \xi}+\phi_{\epsilon}$ is a solution of (1.8) (see for example [18, Proposition 2.2]). Moreover, it is not difficult to check that

$$
\tilde{J}_{\epsilon}(d, \eta)=J_{\epsilon}\left(W_{\delta, \xi}\right)+o\left(|\epsilon|^{3}\right)
$$

uniformly with respect to $(d, \eta)$ which satisfies (2.4) (see for example [18, Proposition 2.2]).

We need only to estimate the main term of the reduced energy $J_{\epsilon}\left(W_{\delta, \xi}\right)$, i.e.,

$$
\begin{aligned}
& J_{\epsilon}\left(u_{0}+\epsilon v_{0}-P U_{\delta, \xi}\right) \\
= & \frac{1}{2} \int_{\Omega}\left|\nabla\left(u_{0}+\epsilon v_{0}-P U_{\delta, \xi}\right)\right|^{2}-\frac{\lambda_{0}+\epsilon}{2} \int_{\Omega}\left(u_{0}+\epsilon v_{0}-P U_{\delta, \xi}\right)^{2}-\frac{1}{3} \int_{\Omega}\left|u_{0}+\epsilon v_{0}-P U_{\delta, \xi}\right|^{3} \\
= & \frac{1}{2} \int_{\Omega}\left|\nabla\left(u_{0}+\epsilon v_{0}\right)\right|^{2}+\frac{1}{2} \int_{\Omega}\left|\nabla P U_{\delta, \xi}\right|^{2}-\frac{\lambda_{0}+\epsilon}{2} \int_{\Omega}\left(u_{0}+\epsilon v_{0}\right)^{2}-\frac{\lambda_{0}+\epsilon}{2} \int_{\Omega}\left(P U_{\delta, \xi}\right)^{2} \\
& -\underbrace{\left(\int_{\Omega} \nabla u_{0} \nabla P U_{\delta, \xi}-\lambda_{0} \int_{\Omega} u_{0} P U_{\delta, \xi}\right)}_{=\int_{\Omega}\left|u_{0}\right| u_{0} P U_{\delta, \xi}}-\epsilon \underbrace{\left(\int_{\Omega} \nabla v_{0} \nabla P U_{\delta, \xi}-\lambda_{0} \int_{\Omega} v_{0} P U_{\delta, \xi}-\int_{\Omega} u_{0} P U_{\delta, \xi}\right)}_{=\int_{\Omega} 2\left|u_{0}\right| v_{0} P U_{\delta, \xi}} \\
& +\epsilon^{2} \int_{\Omega} v_{0} P U_{\delta, \xi}-\frac{1}{3} \int_{\Omega}\left|u_{0}+\epsilon v_{0}-P U_{\delta, \xi}\right|^{3}
\end{aligned}
$$




$$
\begin{aligned}
& =\underbrace{\frac{1}{2} \int_{\Omega}\left|\nabla\left(u_{0}+\epsilon v_{0}\right)\right|^{2}-\frac{\lambda_{0}+\epsilon}{2} \int_{\Omega}\left(u_{0}+\epsilon v_{0}\right)^{2}-\frac{1}{3} \int_{\Omega}\left|u_{0}+\epsilon v_{0}\right|^{3}}_{=: I_{1}} \\
& +\underbrace{\frac{1}{2} \int_{\Omega}\left|\nabla P U_{\delta, \xi}\right|^{2}-\frac{1}{3} \int_{\Omega} P U_{\delta, \xi}^{3}}_{=: I_{2}}-\underbrace{\frac{\lambda_{0}}{2} \int_{\Omega} P U_{\delta, \xi}^{2}+\int_{\Omega} u_{0} P U_{\delta, \xi}^{2}}_{=: I_{3}} \underbrace{-\frac{\epsilon}{2} \int_{\Omega} P U_{\delta, \xi}^{2}+\epsilon \int v_{0} P U_{\delta, \xi}^{2}}_{=: I_{4}} \\
& \underbrace{-\frac{1}{3} \int_{\Omega}\left(\left|u_{0}+\epsilon v_{0}-P U_{\delta, \xi}\right|^{3}-\left|u_{0}+\epsilon v_{0}\right|^{3}-P U_{\delta, \xi}^{3}+3\left(u_{0}+\epsilon v_{0}\right) P U_{\delta, \xi}^{2}+3\left|u_{0}+\epsilon u_{0}\right|\left(u_{0}+\epsilon v_{0}\right) P U_{\delta, \xi}\right)}_{=: I_{5}} \\
& \underbrace{+\int_{\Omega}\left[\left|u_{0}+\epsilon v_{0}\right|\left(u_{0}+\epsilon v_{0}\right)-\left(\left|u_{0}\right| u_{0}+2 \epsilon\left|u_{0}\right| v_{0}\right)\right] P U_{\delta, \xi}}_{=: I_{6}}+\underbrace{\epsilon^{2} \int_{\Omega} v_{0} P U_{\delta, \xi}}_{=: I_{7}} .
\end{aligned}
$$

It is clear that

$$
I_{7}=\mathcal{O}\left(\epsilon^{2} \int_{\Omega} \frac{\delta^{2}}{|x-\xi|^{4}} d x\right)=\mathcal{O}\left(\epsilon^{2} \delta^{2}\right)=\mathcal{O}\left(\epsilon^{4}\right)
$$

To estimate $I_{6}$ by (2.6) it follows that

$$
I_{6}=\mathcal{O}\left(\epsilon^{2} \int_{\Omega} P U_{\delta, \xi}\right)=\mathcal{O}\left(\epsilon^{2} \delta^{2}\right)=\mathcal{O}\left(\epsilon^{4}\right)
$$

Now, $I_{1}$ does not depend neither on $d$ nor on $\eta$ and it will be included in the constant $\mathfrak{c}_{0}$ in (2.7). By (2.2)

$$
\begin{aligned}
I_{2} & =\frac{1}{2} \int_{\Omega} U_{\delta, \xi}^{3}-\frac{1}{3} \int_{\Omega} P U_{\delta, \xi}^{3} \\
& =\frac{1}{2} \int_{\Omega} U_{\delta, \xi}^{3}-\frac{1}{3} \int_{\Omega}\left(U_{\delta, \xi}(x)-\alpha_{6} \delta^{2} H(x, \xi)+\mathcal{O}\left(\delta^{4}\right)\right)^{3} \\
& =\frac{1}{6} \int_{\mathbb{R}^{6}} U^{3}+\mathcal{O}\left(\delta^{2} \int_{\Omega} U_{\delta, \xi}^{2}\right)+O\left(\delta^{4}\right) \\
& =\frac{1}{6} \int_{\mathbb{R}^{6}} U_{\delta, \xi}^{3}+O\left(\delta^{4}\right) .
\end{aligned}
$$

Now, setting

$$
\varphi_{\delta, \xi}:=P U_{\delta, \xi}-U_{\delta, \xi}=\mathcal{O}\left(\delta^{2}\right)
$$

by (2.2) and (2.4)

$$
\begin{aligned}
I_{3} & =\int_{\Omega}\left(u_{0}(x)-\frac{\lambda_{0}}{2}\right)\left(U_{\delta, \xi}+\varphi_{\delta, \xi}\right)^{2} \\
& =\int_{\Omega}\left(u_{0}(x)-u_{0}\left(\xi_{0}\right)\right) U_{\delta, \xi}^{2}+\mathcal{O}\left(\delta^{4}\right) \\
& =\int_{\Omega}\left[\frac{1}{2}\left\langle D^{2} u_{0}\left(\xi_{0}\right)\left(x-\xi_{0}\right),\left(x-\xi_{0}\right)\right\rangle+\mathcal{O}\left(\left|x-\xi_{0}\right|^{3}\right)\right] \alpha_{6}^{2} \frac{\delta^{4}}{\left(\delta^{2}+|x-\xi|^{2}\right)^{4}} d x+\mathcal{O}\left(\delta^{4}\right) \\
& =\alpha_{6}^{2} \int_{\Omega} \frac{1}{2}\left\langle D^{2} u_{0}\left(\xi_{0}\right)\left(x-\xi_{0}\right),\left(x-\xi_{0}\right)\right\rangle \frac{\delta^{4}}{\left(\delta^{2}+|x-\xi|^{2}\right)^{4}} d x+\mathcal{O}\left(\delta^{4}\right)
\end{aligned}
$$




$$
\begin{aligned}
& =\alpha_{6}^{2} \delta^{2} \int_{\frac{\Omega-\xi}{\delta}} \frac{1}{2}\left\langle D^{2} u_{0}\left(\xi_{0}\right)(\delta y+\sqrt{\delta} \eta),(\delta y+\sqrt{\delta} \eta)\right\rangle \frac{1}{\left(1+|y|^{2}\right)^{4}} d y+\mathcal{O}\left(\delta^{4}\right) \\
& =\frac{\alpha_{6}^{2}}{2} \delta^{3}\left(\int_{\mathbb{R}^{6}} \frac{1}{\left(1+|y|^{2}\right)^{4}} d y\right)\left\langle D^{2} u_{0}\left(\xi_{0}\right) \eta, \eta\right\rangle+\mathcal{O}\left(\delta^{4}|\ln \delta|\right) \\
& =\frac{\alpha_{6}^{2}}{2} d^{3}|\epsilon|^{3}\left(\int_{\mathbb{R}^{6}} \frac{1}{\left(1+|y|^{2}\right)^{4}} d y\right)\left\langle D^{2} u_{0}\left(\xi_{0}\right) \eta, \eta\right\rangle+\mathcal{O}\left(\epsilon^{4}|\ln | \epsilon||\right),
\end{aligned}
$$

and analogously

$$
\begin{aligned}
I_{4} & =\epsilon \int_{\Omega}\left(v_{0}(x)-\frac{1}{2}\right) P U_{\delta, \xi}^{2} \\
& =\epsilon\left[\alpha_{6}^{2} \delta^{2}\left(\int_{\mathbb{R}^{6}} \frac{1}{\left(1+|y|^{2}\right)^{4}} d y\right)\left(v_{0}\left(\xi_{0}\right)-\frac{1}{2}\right)+o(1)\right] \\
& =\epsilon^{3} d^{2}\left[\alpha_{6}^{2}\left(\int_{\mathbb{R}^{6}} \frac{1}{\left(1+|y|^{2}\right)^{4}} d y\right)\left(v_{0}\left(\xi_{0}\right)-\frac{1}{2}\right)+o(1)\right] .
\end{aligned}
$$

Finally, we have to estimate $I_{5}$.

We point out that

$$
\begin{aligned}
& \left|u_{0}+\epsilon v_{0}-P U_{\delta, \xi}\right|^{3}-\left|u_{0}+\epsilon v_{0}\right|^{3}-P U_{\delta, \xi}^{3} \\
& \quad+3\left(u_{0}+\epsilon v_{0}\right) P U_{\delta, \xi}^{2}+3\left|u_{0}+\epsilon u_{0}\right|\left(u_{0}+\epsilon v_{0}\right) P U_{\delta, \xi}=0 \text { if } u_{0}+\epsilon v_{0} \leq 0
\end{aligned}
$$

and so

$$
\begin{aligned}
I_{5}=- & \frac{1}{3} \int_{\left\{u_{0}+\epsilon v_{0} \geq 0\right\}}\left(\left|u_{0}+\epsilon v_{0}-P U_{\delta, \xi}\right|^{3}-\left(u_{0}+\epsilon v_{0}\right)^{3}-P U_{\delta, \xi}^{3}\right. \\
& \left.+3\left(u_{0}+\epsilon v_{0}\right) P U_{\delta, \xi}^{2}+3\left(u_{0}+\epsilon v_{0}\right)^{2} P U_{\delta, \xi}\right) d x \\
=- & \frac{1}{3} \int_{\left\{u_{0}+\epsilon v_{0} \geq P U_{\delta, \xi}\right\}}\left(-2 P U_{\delta, \xi}^{3}+6\left(u_{0}+\epsilon v_{0}\right) P U_{\delta, \xi}^{2}\right) \\
& -\frac{1}{3} \int_{\left\{0<u_{0}+\epsilon v_{0}<P U_{\delta, \xi}\right\}}\left(-2\left(u_{0}+\epsilon v_{0}\right)^{3}+6\left(u_{0}+\epsilon v_{0}\right)^{2} P U_{\delta, \xi}\right) .
\end{aligned}
$$

First of all we claim that for any $\sigma>0$ there exists $\varepsilon_{0}>0$ such that for any $\epsilon \in$ $\left(-\varepsilon_{0}, \varepsilon_{0}\right)$ and $(d, \xi)$ satisfying $(2.4)$

$$
B\left(\xi, R_{\delta}^{1} \sqrt{\delta}\right) \subset\left\{x \in \Omega: 0<u_{0}(x)+\epsilon v_{0}(x)<P U_{\delta, \xi}(x)\right\} \cap B\left(\xi, \delta^{\frac{1}{4}}\right) \subset B\left(\xi, R_{\delta}^{2} \sqrt{\delta}\right),
$$

where

$$
R_{\delta}^{1}, R_{\delta}^{2}=R_{0}+o(1) \quad \text { with } R_{0}:=\left(\frac{\alpha_{6}}{u_{0}\left(\xi_{0}\right)}\right)^{\frac{1}{4}} .
$$

We remind that $\delta=\mathcal{O}(\epsilon)$ and also that

$$
P U_{\delta, \xi}(x)=\alpha_{6} \frac{\delta^{2}}{\left(\delta^{2}+|x-\xi|^{2}\right)^{2}}+\mathcal{O}\left(\epsilon^{2}\right)
$$


uniformly in $\Omega$. If $|x-\xi|<R_{\delta}^{1} \sqrt{\delta}$ is small enough then by mean value theorem $u_{0}(x)+$ $\epsilon v_{0}(x)=u_{0}\left(\xi_{0}\right)+\mathcal{O}_{1}(\epsilon)$ and

$$
\begin{aligned}
u_{0}(x)+\epsilon v_{0}(x)<P U_{\delta, \xi}(x) & \Leftrightarrow \frac{u_{0}\left(\xi_{0}\right)}{\alpha_{6}}+\mathcal{O}_{1}(\epsilon)<\frac{\delta^{2}}{\left(\delta^{2}+|x-\xi|^{2}\right)^{2}} \\
& \Leftrightarrow|x-\xi| \leq \sqrt{\delta} \underbrace{\left(\frac{1}{\left(\frac{u_{0}\left(\xi_{0}\right)}{\alpha_{6}}+\mathcal{O}_{1}(\epsilon)\right)^{\frac{1}{2}}}-\delta\right)^{\frac{1}{2}}}_{R_{\delta}^{1}},
\end{aligned}
$$

and the first inclusion in (2.9) together with (2.10) follow. On the other hand, again by mean value theorem we have

$$
u_{0}(x)+\epsilon v_{0}(x)=u_{0}\left(\xi_{0}\right)+\mathcal{O}_{2}(\sqrt{\delta})
$$

for any $x \in B\left(\xi, \delta^{\frac{1}{4}}\right)$ and arguing as above we get the second inclusion in (2.9) and (2.10). It is useful to point out that by (2.9) we immediately get

$$
B^{c}\left(\xi, R_{\delta}^{1} \sqrt{\delta}\right) \supset\left\{x \in \Omega: u_{0}(x)+\epsilon v_{0}(x) \geq P U_{\delta, \xi}(x)\right\} \cup B^{c}\left(\xi, \delta^{\frac{1}{4}}\right) \supset B^{c}\left(\xi, R_{\delta}^{2} \sqrt{\delta}\right) .
$$

Now by (2.9) and (2.11) we deduce

$$
\begin{aligned}
I_{5}= & -\frac{1}{3} \int_{\left\{u_{0}+\epsilon v_{0} \geq P U_{\delta, \xi}\right\}}\left(-2 P U_{\delta, \xi}^{3}+6\left(u_{0}+\epsilon v_{0}\right) P U_{\delta, \xi}^{2}\right) \\
& -\frac{1}{3} \int_{\left\{0<u_{0}+\epsilon v_{0}<P U_{\delta, \xi}\right\}}\left(-2\left(u_{0}+\epsilon v_{0}\right)^{3}+6\left(u_{0}+\epsilon v_{0}\right)^{2} P U_{\delta, \xi}\right) \\
= & -\frac{1}{3} \int_{\left\{u_{0}+\epsilon v_{0} \geq P U_{\delta, \xi}\right\} \cup B^{c}\left(\xi, \delta \frac{1}{4}\right)}\left(-2 P U_{\delta, \xi}^{3}+6\left(u_{0}+\epsilon v_{0}\right) P U_{\delta, \xi}^{2}\right) \\
& +\frac{1}{3} \int_{B^{c}\left(\xi, \delta \frac{1}{4}\right) \backslash\left\{u_{0}+\epsilon v_{0} \geq P U_{\delta, \xi}\right\} \cap B^{c}\left(\xi, \delta \frac{1}{4}\right)}\left(-2 P U_{\delta, \xi}^{3}+6\left(u_{0}+\epsilon v_{0}\right) P U_{\delta, \xi}^{2}\right) \\
& -\frac{1}{3} \int_{\left\{0<u_{0}+\epsilon v_{0}<P U_{\delta, \xi}\right\} \cap B\left(\xi, \delta^{\frac{1}{4}}\right)}\left(-2\left(u_{0}+\epsilon v_{0}\right)^{3}+6\left(u_{0}+\epsilon v_{0}\right)^{2} P U_{\delta, \xi}\right) \\
& -\frac{1}{3} \int_{\left\{0<u_{0}+\epsilon v_{0}<P U_{\delta, \xi}\right\} \cap B^{c}\left(\xi, \delta^{\frac{1}{4}}\right)}\left(-2\left(u_{0}+\epsilon v_{0}\right)^{3}+6\left(u_{0}+\epsilon v_{0}\right)^{2} P U_{\delta, \xi}\right) \\
= & -\frac{1}{3} \int_{\left\{u_{0}+\epsilon v_{0} \geq P U_{\delta, \xi}\right\} \cup B^{c}\left(\xi, \delta^{\frac{1}{4}}\right)}\left(-2 P U_{\delta, \xi}^{3}+6\left(u_{0}+\epsilon v_{0}\right) P U_{\delta, \xi}^{2}\right) \\
& -\frac{1}{3} \int_{\left\{0<u_{0}+\epsilon v_{0}<P U_{\delta, \xi}\right\} \cap B\left(\xi, \delta^{\frac{1}{4}}\right)}\left(-2\left(u_{0}+\epsilon v_{0}\right)^{3}+6\left(u_{0}+\epsilon v_{0}\right)^{2} P U_{\delta, \xi}\right)+o\left(\delta^{3}\right),
\end{aligned}
$$


because

$$
\begin{aligned}
& \int_{B^{c}\left(\xi, \delta^{\frac{1}{4}}\right) \backslash\left\{u_{0}+\epsilon v_{0} \geq P U_{\delta, \xi}\right\} \cap B^{c}\left(\xi, \delta^{\frac{1}{4}}\right)}\left(-2 P U_{\delta, \xi}^{3}+6\left(u_{0}+\epsilon v_{0}\right) P U_{\delta, \xi}^{2}\right) \\
& =\mathcal{O}\left(\int_{B^{c}\left(\xi, \delta \frac{1}{4}\right)}\left(U_{\delta, \xi}^{3}+U_{\delta, \xi}^{2}\right)\right)=\mathcal{O}\left(\delta^{\frac{7}{2}}\right), \\
& \int_{\left\{0<u_{0}+\epsilon v_{0}<P U_{\delta, \xi}\right\} \cap B^{c}\left(\xi, \delta \frac{1}{4}\right)}\left(-2\left(u_{0}+\epsilon v_{0}\right)^{3}+6\left(u_{0}+\epsilon v_{0}\right)^{2} P U_{\delta, \xi}\right) \\
& =\mathcal{O}\left(\delta^{3} \operatorname{meas}\left\{0<u_{0}(x)<2 \delta\right\}\right)=o\left(\delta^{3}\right),
\end{aligned}
$$

since

$$
\begin{array}{lc}
P U_{\delta, \xi}(x)=\mathcal{O}(\delta) & \text { if }|x-\xi| \geq \delta^{\frac{1}{4}}, \\
\left\{0<u_{0}+\epsilon v_{0}<P U_{\delta, \xi}\right\} \cap B^{c}\left(\xi, \delta^{\frac{1}{4}}\right) \subset\left\{0<u_{0}(x)<2 \delta\right\} & \text { if } \delta \text { is small enough. }
\end{array}
$$

Next we claim that

$$
\begin{aligned}
- & \frac{1}{3} \int_{\left\{u_{0}+\epsilon v_{0} \geq P U_{\delta, \xi}\right\} \cup B^{c}\left(\xi, \delta^{\frac{1}{4}}\right)}\left(-2 P U_{\delta, \xi}^{3}+6\left(u_{0}+\epsilon v_{0}\right) P U_{\delta, \xi}^{2}\right) \\
& -\frac{1}{3} \int_{\left\{0<u_{0}+\epsilon v_{0}<P U_{\delta, \xi}\right\} \cap B\left(\xi, \delta^{\frac{1}{4}}\right)}\left(-2\left(u_{0}+\epsilon v_{0}\right)^{3}+6\left(u_{0}+\epsilon v_{0}\right)^{2} P U_{\delta, \xi}\right)+o\left(\delta^{3}\right) \\
=- & \frac{1}{3} \int_{\left\{u_{0}+\epsilon v_{0} \geq P U_{\delta, \xi}\right\} \cup B^{c}\left(\xi, \delta^{\frac{1}{4}}\right)}\left(-2 P U_{\delta, \xi}^{3}+6 u_{0} P U_{\delta, \xi}^{2}\right) \\
& -\frac{1}{3} \int_{\left\{0<u_{0}+\epsilon v_{0}<P U_{\delta, \xi}\right\} \cap B\left(\xi, \delta^{\frac{1}{4}}\right)}\left(-2 u_{0}^{3}+6 u_{0}^{2} P U_{\delta, \xi}\right)+o\left(\delta^{3}\right) .
\end{aligned}
$$

Indeed using (2.11) and (2.9) we get

$$
\int_{\left\{u_{0}+\epsilon v_{0} \geq P U_{\delta, \xi}\right\} \cup B^{c}\left(\xi, \delta^{\frac{1}{4}}\right)} P U_{\delta, \xi}^{2}=\mathcal{O}\left(\int_{B^{c}\left(\xi, \delta^{\frac{1}{2}}\right)} U_{\delta, \xi}^{2}\right)=\mathcal{O}\left(\delta^{3}\right),
$$

$\operatorname{meas} B\left(\xi, \delta^{\frac{1}{2}}\right)=\mathcal{O}\left(\delta^{3}\right)$ and

$$
\int_{\left\{u_{0}+\epsilon v_{0}<P U_{\delta, \xi}\right\} \cap B\left(\xi, \delta^{\frac{1}{4}}\right)} P U_{\delta, \xi}=\mathcal{O}\left(\int_{B\left(\xi, \delta^{\frac{1}{2}}\right)} U_{\delta, \xi}\right)=\mathcal{O}\left(\delta^{3}\right) .
$$

We estimate the last two terms in the expansion of $I_{5}$. By (2.11)

$$
B^{c}\left(\xi, R_{\delta}^{2} \sqrt{\delta}\right) \subset\left\{x \in \Omega: u_{0}(x)+\epsilon v_{0}(x) \geq P U_{\delta, \xi}\right\} \cup B^{c}\left(\xi, \delta^{\frac{1}{4}}\right) \subset B^{c}\left(\xi, R_{\delta}^{1} \sqrt{\delta}\right) .
$$


Hence

$$
\begin{aligned}
& \int_{|x-\xi|>R_{\delta}^{2} \sqrt{\delta}}\left(-2 P U_{\delta, \xi}^{3}+6 u_{0} P U_{\delta, \xi}^{2}\right) \\
\leq & \int_{\left\{u_{0}+\epsilon v_{0} \geq P U_{\delta, \xi}\right\} \cup B\left(\xi, \delta \frac{1}{4}\right)}\left(-2 P U_{\delta, \xi}^{3}+6 u_{0} P U_{\delta, \xi}^{2}\right) \\
\leq & \int_{|x-\xi|>R_{\delta}^{1} \sqrt{\delta}}\left(-2 P U_{\delta, \xi}^{3}+6 u_{0} P U_{\delta, \xi}^{2}\right) .
\end{aligned}
$$

Now if $R_{\delta}$ denotes either $R_{\delta}^{1}$ or $R_{\delta}^{2}$ we get

$$
\begin{aligned}
& \int_{|x-\xi|>R_{\delta} \sqrt{\delta}}\left(-2 P U_{\delta, \xi}^{3}+6 u_{0} P U_{\delta, \xi}^{2}\right) \\
= & -2 \int_{|x-\xi|>R_{\delta} \sqrt{\delta}} U_{\delta, \xi}^{3}+6 \int_{|x-\xi|>R_{\delta} \sqrt{\delta}} u_{0} U_{\delta, \xi}^{2}+\mathcal{O}\left(\delta^{4}\right) \\
= & -2 \int_{|y|>\frac{R_{\delta}}{\sqrt{\delta}}} \frac{\alpha_{6}^{3}}{\left(1+|y|^{2}\right)^{6}}+6 \delta^{2} \int_{|y|>\frac{R_{\delta}}{\sqrt{\delta}}} u_{0}(\delta y+\xi) \frac{\alpha_{6}^{2}}{\left(1+|y|^{2}\right)^{4}}+\mathcal{O}\left(\delta^{4}\right) \\
=- & 2 \omega_{6} \alpha_{6}^{3} \int_{\frac{R_{\delta}}{\sqrt{\delta}}}^{+\infty} \frac{r^{5}}{\left(1+r^{2}\right)^{6}}+6 \delta^{2} \omega_{6} \alpha_{6}^{2} u_{0}\left(\xi_{0}\right) \int_{\frac{R_{\delta}}{\sqrt{\delta}}}^{+\infty} \frac{r^{5}}{\left(1+r^{2}\right)^{4}} \\
& +\mathcal{O}\left(\delta^{4} \int_{\frac{R_{\delta}}{\sqrt{\delta}}}^{+\infty} \frac{r^{7}}{\left(1+r^{2}\right)^{4}}\right)+\mathcal{O}\left(\delta^{4}\right) \\
=- & \frac{1}{3} \omega_{6} \alpha_{6}^{3} R_{\delta}^{-6} \delta^{3}+3 \delta^{3} \omega_{6} \alpha_{6}^{2} R_{\delta}^{-2} u_{0}\left(\xi_{0}\right)+\mathcal{O}\left(\delta^{4}|\log \delta|\right) \\
= & -\frac{1}{3} \omega_{6} \alpha_{6}^{3} R_{0}^{-6} \delta^{3}+3 \delta^{3} \omega_{6} \alpha_{6}^{2} R_{0}^{-2} u_{0}\left(\xi_{0}\right)+o\left(\delta^{3}\right) \quad \text { because of (2.10) }
\end{aligned}
$$

and by comparison

$$
\begin{aligned}
& \int_{\left\{u_{0}+\epsilon v_{0} \geq P U_{\delta, \xi}\right\} \cup B^{c}\left(\xi, \delta^{\frac{1}{4}}\right)}\left(-2 P U_{\delta, \xi}^{3}+6 u_{0} P U_{\delta, \xi}^{2}\right) \\
= & -\frac{1}{3} \omega_{6} \alpha_{6}^{3}\left(R_{0}\right)^{-6} \delta^{3}+3 \delta^{3} \omega_{6} \alpha_{6}^{2}\left(R_{0}\right)^{-2} u_{0}\left(\xi_{0}\right)+o\left(\delta^{3}\right) .
\end{aligned}
$$

In a similar way, by (2.9)

$$
\begin{aligned}
& \int_{|x-\xi|<R_{\delta}^{1} \sqrt{\delta}}\left(-2 u_{0}^{3}+6 u_{0}^{2} P U_{\delta, \xi}\right) \\
\leq & \int_{\left\{0<u_{0}+\epsilon v_{0}<P U_{\delta, \xi}\right\} \cap B(\xi, \delta)}\left(-2 u_{0}^{3}+6 u_{0}^{2} P U_{\delta, \xi}\right) \\
\leq & \int_{|x-\xi|<R_{\delta}^{2} \sqrt{\delta}}\left(-2 u_{0}^{3}+6 u_{0}^{2} P U_{\delta, \xi}\right),
\end{aligned}
$$


and if $R_{\delta}$ denotes either $R_{\delta}^{1}$ or $R_{\delta}^{2}$ we get

$$
\begin{aligned}
& \int_{|x-\xi|<R_{\delta} \sqrt{\delta}}\left(-2 u_{0}^{3}+6 u_{0}^{2} P u_{\delta, \xi}\right) \\
= & -2 \delta^{6} \int_{|y|<\frac{R_{\delta}}{\sqrt{\delta}}} u_{0}^{3}(\delta y+\xi)+6 \delta^{4} \int_{|y|<\frac{R_{\delta}}{\sqrt{\delta}}} u_{0}^{2}(\delta y+\xi) \frac{\alpha_{6}}{\left(1+|y|^{2}\right)^{2}}+\mathcal{O}\left(\delta^{5}\right) \\
= & \left(-2 u_{0}^{3}\left(\xi_{0}\right)+\mathcal{O}(\sqrt{\delta})\right) \delta^{6} \omega_{6} \int_{0}^{\frac{R_{\delta}}{\sqrt{\delta}}} r^{5} \\
& +6 \alpha_{6}\left(u_{0}^{2}\left(\xi_{0}\right)+\mathcal{O}(\sqrt{\delta})\right) \delta^{4} \omega_{6} \int_{0}^{\frac{R_{\delta}}{\sqrt{\delta}}} \frac{r^{5}}{\left(1+r^{2}\right)^{2}}+\mathcal{O}\left(\delta^{5}\right) \\
= & -2 \delta^{3} u_{0}^{3}\left(\xi_{0}\right) \omega_{6} R_{\delta}^{6}+3 \alpha_{6} \delta^{3} u_{0}^{2}\left(\xi_{0}\right) \omega_{6} R_{\delta}^{2}+\mathcal{O}\left(\delta^{\frac{7}{2}}\right) \\
= & -2 \delta^{3} u_{0}^{3}\left(\xi_{0}\right) \omega_{6} R_{0}^{6}+3 \alpha_{6} \delta^{3} u_{0}^{2}\left(\xi_{0}\right) \omega_{6} R_{0}^{2}+o\left(\delta^{3}\right) \quad \text { because of }(2.10),
\end{aligned}
$$

and by comparison

$$
\begin{aligned}
& \int_{\left\{u_{0}+\epsilon v_{0}<P U_{\delta, \xi}\right\} \cap B\left(\xi, \delta^{\frac{1}{4}}\right)}\left(-2 u_{0}^{3}+6 u_{0}^{2} P U_{\delta, \xi}\right) \\
= & -2 \delta^{3} u_{0}^{3}\left(\xi_{0}\right) \omega_{6} R_{0}^{6}+3 \alpha_{6} \delta^{3} u_{0}^{2}\left(\xi_{0}\right) \omega_{6} R_{0}^{2}+o\left(\delta^{3}\right) .
\end{aligned}
$$

Finally, by (2.13) and (2.12)

$$
I_{5}=|\epsilon|^{3} d^{3}\left(-\frac{11}{9} \omega_{6} \alpha_{6}^{\frac{3}{2}}\left(u_{0}\left(\xi_{0}\right)\right)^{\frac{3}{2}}+o(1)\right) .
$$

Collecting all the previous estimates we get

$$
\tilde{J}_{\epsilon}(d, \eta)=\mathfrak{c}_{0}(\epsilon)+|\epsilon|^{3} \underbrace{\left\{\operatorname{sgn}(\epsilon)\left(1-2 v_{0}\left(\xi_{0}\right)\right) d^{2} \mathfrak{a}_{1}+d^{3}\left(\mathfrak{a}_{2}\left\langle D^{2} u_{0}\left(\xi_{0}\right) \eta, \eta\right\rangle-\mathfrak{a}_{3}\right)\right\}}_{=: Y(d, \eta)}+o\left(|\epsilon|^{3}\right)
$$

with

$$
\begin{aligned}
& \mathfrak{a}_{1}=\alpha_{6}^{2}\left(\int_{\mathbb{R}^{6}} \frac{1}{\left(1+|y|^{2}\right)^{4}} d y\right)=96 \omega_{6}, \\
& \mathfrak{a}_{2}=\frac{\alpha_{6}^{2}}{2} \int_{\mathbb{R}^{6}} \frac{d y}{\left(1+|y|^{2}\right)^{4}} \\
& \mathfrak{a}_{3}=\frac{11}{9} \omega_{6} \alpha_{6}^{\frac{3}{2}}\left(u_{0}\left(\xi_{0}\right)\right)^{\frac{3}{2}},
\end{aligned}
$$

and that concludes the proof.

We are now in position to prove Theorem 1.1. 
Proof of Theorem 1.1. The claim follows by Proposition 2.2 taking into account that if

$$
\operatorname{sgn}(\epsilon)\left(1-2 v_{0}\left(\xi_{0}\right)\right)>0
$$

the function $\mathrm{Y}$ has always an isolated maximum point $\left(d_{0}, 0\right)$, with

$$
d_{0}:=\frac{2 \mathfrak{a}_{1}}{3 \mathfrak{a}_{3}} \operatorname{sgn}(\epsilon)\left(1-2 v_{0}\left(\xi_{0}\right)\right),
$$

which is stable under uniform perturbations.

\section{A generic result}

Let $\Omega_{0}$ be a bounded and smooth domain in $\mathbb{R}^{n}$, we let $D$ be and open neighbourhood of $\overline{\Omega_{0}}$ and $\alpha \in(0,1)$. There exists $\epsilon>0$ such that if $\theta \in C^{3, \alpha}\left(\bar{D}, \mathbb{R}^{n}\right)$ with $\|\theta\|_{2, \alpha} \leq \epsilon$ then $\Theta=I+\theta$ maps $\Omega_{0}$ in a one-to-one way onto the smooth domain $\Omega_{\theta}:=\Theta\left(\Omega_{0}\right)$ with boundary $\partial \Omega_{\theta}=\Theta\left(\partial \Omega_{0}\right)$. If $x \in \Omega_{0}$ we agree that $\hat{x}=\Theta x=(I+\theta) x \in \Omega_{\theta}$. If $\hat{u} \in H_{0}^{1}\left(\Omega_{\theta}\right) \cap H^{2}\left(\Omega_{\theta}\right)$ then it is clear that $u=\hat{u} \circ \Theta \in H_{0}^{1}\left(\Omega_{0}\right) \cap H^{2}\left(\Omega_{0}\right)$.

Our result reads as follows.

Theorem 3.1. The set

$$
\begin{aligned}
\Xi:=\left\{\theta \in C^{3, \alpha}\left(\bar{D}, \mathbb{R}^{n}\right):\right. & \text { if } \lambda>0 \text { and } u \in H_{0}^{1}\left(\Omega_{\theta}\right) \text { solve } \\
& \Delta u+\lambda u+|u|^{\frac{4}{n-2}} u=0 \text { in } \Omega_{\theta}, u=0 \text { on } \partial \Omega_{\theta}, \\
& \text { then } u \text { is non-degenerate }\}
\end{aligned}
$$

is a residual subset in $C^{3, \alpha}\left(\bar{D}, \mathbb{R}^{n}\right)$, i.e., $C^{3, \alpha}\left(\bar{D}, \mathbb{R}^{n}\right) \backslash \Xi$ is a countable union of close subsets without interior points.

The proof relies on the following abstract transversality theorem (see $[23,26,29])$.

Theorem 3.2. Let $X, Y, Z$ be three Banach spaces and $U \subset X, V \subset Y$ open subsets. Let $F: U \times V \rightarrow Z$ be a $C^{\alpha}$-map with $\alpha \geq 1$. Assume that

i) for any $y \in V, F(\cdot, y): U \rightarrow Z$ is a Fredholm map of index $l$ with $l \leq \alpha$;

ii) 0 is a regular value of $F$, i.e., the operator $F^{\prime}\left(x_{0}, y_{0}\right): X \times Y \rightarrow Z$ is onto at any point $\left(x_{0}, y_{0}\right)$ such that $F\left(x_{0}, y_{0}\right)=0$;

iii) the map $\pi \circ i: F^{-1}(0) \rightarrow Y$ is $\sigma$-proper, i.e., $F^{-1}(0)=\cup_{s=1}^{+\infty} C_{s}$ where $C_{s}$ is a closed set and the restriction $\pi \circ i_{\left.\right|_{C_{s}}}$ is proper for any s; here $i: F^{-1}(0) \rightarrow Y$ is the canonical embedding and $\pi: X \times Y \rightarrow Y$ is the projection.

Then the set $\mathcal{V}:=\{y \in V: 0$ is a regular value of $F(\cdot, y)\}$ is a residual subset of $V$, i.e., $V \backslash \mathcal{V}$ is a countable union of close subsets without interior points. 
Indeed, in our case we choose

$$
\begin{aligned}
& X=\mathbb{R} \times\left(H_{0}^{1}\left(\Omega_{0}\right) \cap H^{2}\left(\Omega_{0}\right)\right), \\
& U=(0, \infty) \times\left(H_{0}^{1}\left(\Omega_{0}\right) \cap H^{2}\left(\Omega_{0}\right) \backslash\{0\}\right), \\
& Y=C^{3, \alpha}\left(\bar{D}, \mathbb{R}^{n}\right) \\
& V=\mathcal{B}_{\epsilon}:=\left\{\theta \in C^{3, \alpha}\left(\bar{D}, \mathbb{R}^{n}\right):\|\theta\|_{3, \alpha}<\epsilon\right\}, \\
& Z=\mathbb{R} \times L^{2}\left(\Omega_{0}\right) .
\end{aligned}
$$

$X$ and $Z$ are Banach spaces equipped with the norms $\|(a, u)\|_{X}:=|a|+\|u\|_{H_{0}^{1} \cap H^{2}\left(\Omega_{0}\right)}$, and $\|(a, u)\|_{Z}:=|a|+\|u\|_{L^{2}\left(\Omega_{0}\right)}$, respectively. Moreover, the function $F: U \times V \rightarrow Z$ is defined by

$$
F(\lambda, u, \theta):=\left(Q(\lambda, \hat{u}, \theta), \Delta_{\hat{x}} \hat{u}+|\hat{u}|^{p-1} \hat{u}+\lambda \hat{u}\right),
$$

where

$$
Q(\lambda, \hat{u}, \theta):=\int_{\Omega_{\theta}}\left(\left|\nabla_{\hat{x}} \hat{u}\right|^{2}-|\hat{u}|^{p+1}-\lambda \hat{u}^{2}\right) d \hat{x}
$$

It is clear that

$$
F(\lambda, u, \theta)=(0,0) \Leftrightarrow \Delta_{\hat{x}} \hat{u}+|\hat{u}|^{p-1} \hat{u}+\lambda \hat{u}=0 \quad \text { in } \Omega_{\theta}, \quad \hat{u}=0 \quad \text { on } \partial \Omega_{\theta} .
$$

Theorem 3.1 will follow by Theorem 3.2 as soon as we prove that $F$ satisfies the assumptions and this is done below.

First of all, we rewrite $F$ in terms of the $x$-variable (see $[21,26]$ )

Lemma 3.1. We have

$$
\begin{aligned}
& Q(\lambda, \hat{u}, \theta):=\int_{\Omega_{0}}\left\{\nabla u \cdot\left[\left(\operatorname{det} \Theta^{\prime}\right)\left(\Theta^{\prime}\right)^{-1}\left({ }^{t} \Theta^{\prime}\right)^{-1} \nabla u\right]-\left(|u|^{p+1}+\lambda u^{2}\right)\left(\operatorname{det} \Theta^{\prime}\right)\right\} d x, \\
& \Delta_{\hat{x}} \hat{u}+|\hat{u}|^{p-1} \hat{u}+\lambda \hat{u}=\operatorname{div}\left[\left(\operatorname{det} \Theta^{\prime}\right)\left(\Theta^{\prime}\right)^{-1}\left({ }^{t} \Theta^{\prime}\right)^{-1} \nabla u\right]+\left(|u|^{p-1} u+\lambda u\right)\left(\operatorname{det} \Theta^{\prime}\right) .
\end{aligned}
$$

At this point it is useful to point out the following fact.

Remark 3.1. We can choose $\epsilon>0$ small enough so that for any $\theta \in \mathcal{B}_{\epsilon}$

$$
\left(\int_{\Omega_{0}}\left(\left|\left\langle\left(\operatorname{det} \Theta^{\prime}\right)\left(\Theta^{\prime}\right)^{-1}\left({ }^{t} \Theta^{\prime}\right)^{-1} \nabla u, \nabla u\right\rangle\right|^{2}+\left|\operatorname{div}\left[\left(\operatorname{det} \Theta^{\prime}\right)\left(\Theta^{\prime}\right)^{-1}\left({ }^{t} \Theta^{\prime}\right)^{-1} \nabla u\right]\right|^{2}\right) d x\right)^{1 / 2}
$$

defines on $H_{0}^{1}\left(\Omega_{0}\right) \cap H^{2}\left(\Omega_{0}\right)$ a norm which is equivalent to the standard one

$$
\|u\|_{H_{0}^{1} \cap H^{2}\left(\Omega_{0}\right)}=\left(\int_{\Omega_{0}}\left(|\nabla u|^{2}+|\Delta u|^{2}\right) d x\right)^{1 / 2} .
$$

Next, we check the differentiability of $F$ (see $[21,26])$. 
Lemma 3.2. The function $F$ is differentiable at any $\left(\lambda_{0}, u_{0}, \theta_{0}\right) \in U \times V$ such that $F\left(\lambda_{0}, u_{0}, \theta_{0}\right)=$ $(0,0)$. Moreover if $\Theta_{0}=I+\theta_{0}$,

$$
\begin{aligned}
F^{\prime}\left(\lambda_{0}, u_{0}, \theta_{0}\right)[\lambda, u]=( & \int_{\Omega_{0}}\left\{2 \nabla u_{0} \cdot\left[\left(\operatorname{det} \Theta_{0}^{\prime}\right)\left(\Theta_{0}^{\prime}\right)^{-1}\left({ }^{t} \Theta_{0}^{\prime}\right)^{-1} \nabla u\right]\right. \\
& \left.-\left((p+1)\left|u_{0}\right|^{p-1} u_{0}+2 \lambda_{0} u_{0}\right) u\left(\operatorname{det} \Theta_{0}^{\prime}\right)\right\} d x-\lambda \int_{\Omega_{0}} u_{0}^{2}\left(\operatorname{det} \Theta_{0}^{\prime}\right) d x \\
& \operatorname{div}\left[\left(\operatorname{det} \Theta_{0}^{\prime}\right)\left(\Theta_{0}^{\prime}\right)^{-1}\left({ }^{t} \Theta_{0}^{\prime}\right)^{-1} \nabla u\right] \\
& \left.+\left(p\left|u_{0}\right|^{p-1}+\lambda_{0}\right) u\left(\operatorname{det} \Theta_{0}^{\prime}\right)+\lambda u_{0}\left(\operatorname{det} \Theta_{0}^{\prime}\right)\right)
\end{aligned}
$$

and if $\theta_{0}=0$,

$$
\begin{aligned}
& F^{\prime}\left(\lambda_{0}, u_{0}, \theta_{0}\right)[\theta] \\
= & \left(\int_{\Omega_{0}}\left\{\nabla u_{0} \cdot\left[(\operatorname{div} \theta) \nabla u_{0}-\left(\theta^{\prime}+{ }^{t} \theta^{\prime}\right) \nabla u_{0}\right]-\left(\left|u_{0}\right|^{p+1}+\lambda_{0} u_{0}^{2}\right)(\operatorname{div} \theta)\right\} d x,\right. \\
& \left.\quad \operatorname{div}\left[(\operatorname{div} \theta) \nabla u_{0}-\left(\theta^{\prime}+{ }^{t} \theta^{\prime}\right) \nabla u_{0}\right]+\left(\left|u_{0}\right|^{p-1} u_{0}+\lambda_{0} u_{0}\right)(\operatorname{div} \theta)\right) .
\end{aligned}
$$

Let us check assumption i) of Theorem 3.2.

Lemma 3.3. For any $\theta \in V$ the function $F(\cdot, \cdot, \theta)$ is a Fredholm map from $U$ into $Z$ of index 0 .

Proof. The partial derivative $F_{\lambda, u}^{\prime}\left(\lambda_{0}, u_{0}, \theta_{0}\right): X \rightarrow Z$ is the sum of an isomorphism $\mathcal{I}$ and a compact perturbation $\mathcal{K}$, namely

$$
\begin{aligned}
& \mathcal{I}(\lambda, u):=\left(-\lambda \int_{\Omega_{0}} u_{0}^{2}\left(\operatorname{det} \Theta_{0}^{\prime}\right) d x, \operatorname{div}\left[\left(\operatorname{det} \Theta_{0}^{\prime}\right)\left(\Theta_{0}^{\prime}\right)^{-1}\left({ }^{t} \Theta_{0}^{\prime}\right)^{-1} \nabla u\right]\right), \\
& \mathcal{K}(\lambda, u):=\left(\int _ { \Omega _ { 0 } } \left\{2 \nabla u_{0} \cdot\left[\left(\operatorname{det} \Theta_{0}^{\prime}\right)\left(\Theta_{0}^{\prime}\right)^{-1}\left({ }^{t} \Theta_{0}^{\prime}\right)^{-1} \nabla u\right]\right.\right. \\
&\left.-\left((p+1)\left|u_{0}\right|^{p-1} u_{0}+2 \lambda_{0} u_{0}\right) u\left(\operatorname{det} \Theta_{0}^{\prime}\right)\right\} d x, \\
&\left.\left(p\left|u_{0}\right|^{p-1}+\lambda_{0}\right) u\left(\operatorname{det} \Theta_{0}^{\prime}\right)+\lambda u_{0}\left(\operatorname{det} \Theta_{0}^{\prime}\right)\right) .
\end{aligned}
$$

Thus, we completed the proof.

Let us check assumption iii) of Theorem 3.2.

Lemma 3.4. The map $\pi \circ i: F^{-1}(0) \rightarrow Y$ is $\sigma$-proper.

Proof. Let us write

$$
F^{-1}(0,0)=\cup_{m=1}^{\infty} \mathcal{C}_{m}, \quad \mathcal{C}_{m}=\left(A_{m} \times B_{m} \times C_{m}\right) \cap F^{-1}(0,0),
$$


where

$$
\begin{aligned}
& A_{m}:=\left\{\frac{1}{m} \leq \lambda \leq m\right\}, \\
& B_{m}:=\left\{u \in H_{0}^{1}\left(\Omega_{0}\right) \cap H^{2}\left(\Omega_{0}\right): \frac{1}{m} \leq\|u\|:=\left(\int_{\Omega_{0}}\left(|\nabla u|^{2}+(\Delta u)^{2}\right) d x\right)^{\frac{1}{2}} \leq m\right\}, \\
& C_{m}:=\left\{\theta \in C^{3, \alpha}\left(\Omega_{0}\right):\|\theta\|_{3, \alpha} \leq \epsilon\left(1-\frac{1}{m}\right)\right\} .
\end{aligned}
$$

Let us fix $m$. We have to prove that if $\left(\theta_{k}\right)_{k \geq 1} \subset C_{m}$ with $\theta_{k} \rightarrow \theta$ and $\left(\lambda_{k}, u_{k}\right)_{k \geq 1} \subset$ $A_{m} \times B_{m}$ is such that $F\left(\lambda_{k}, u_{k}, \theta_{k}\right)=0$ then, up to a subsequence, $\left(\lambda_{k}, u_{k}\right) \rightarrow(\lambda, u) \in$ $A_{m} \times B_{m}$ and $F(\lambda, u, \theta)=0$. First of all, up to a subsequence, we have $\lambda_{k} \rightarrow \lambda \in A_{m}$ and $u_{k} \rightarrow u$ weakly in $H_{0}^{1}\left(\Omega_{0}\right) \cap H^{2}\left(\Omega_{0}\right)$ and strongly in $L^{q}\left(\Omega_{0}\right)$ for any $q>1$ if $n=3,4$ and $1<q<\frac{2 n}{n-4}$ if $n \geq 5$. If $\Theta_{k}=I+\theta_{k}$ we know that $\Theta_{k} \rightarrow \Theta:=I+\theta$ in $C^{1, \alpha}\left(\Omega_{0}, \mathbb{R}^{n}\right)$. Now, condition $F\left(\lambda_{k}, u_{k}, \theta_{k}\right)=0$ reads as

$$
\begin{array}{ll}
\operatorname{div}(\underbrace{\left(\operatorname{det} \Theta_{k}^{\prime}\right)\left(\Theta_{k}^{\prime}\right)^{-1}\left(\Theta_{k}^{\prime}\right)^{-1}}_{=A_{k}} \nabla u_{k})+\underbrace{\left(\left|u_{k}\right|^{p-1} u_{k}+\lambda_{k} u_{k}\right)\left(\operatorname{det} \Theta_{k}^{\prime}\right)}_{=f_{k}}=0 & \text { in } \Omega_{0}, \\
u=0 & \text { on } \partial \Omega_{0} .
\end{array}
$$

In particular, for any $\varphi \in H_{0}^{1}\left(\Omega_{0}\right)$

$$
\int_{\Omega_{0}}\left[\left\langle A_{k} \nabla u_{k}, \nabla \varphi\right\rangle+f_{k} \varphi\right] d x=0
$$

and so passing to the limit

$$
\left.\int_{\Omega_{0}}[\langle\underbrace{\left(\operatorname{det} \Theta^{\prime}\right)\left(\Theta^{\prime}\right)^{-1}\left({ }^{t} \Theta^{\prime}\right)^{-1}}_{=A} \nabla u, \nabla \varphi\rangle-\underbrace{\left(|u|^{p-1} u+\lambda u\right)\left(\operatorname{det} \Theta^{\prime}\right.}_{=f}) \varphi\right] d x=0,
$$

namely

$$
\operatorname{div}(A \nabla u)+f=0 \quad \text { in } \Omega_{0}, \quad u=0 \quad \text { on } \partial \Omega_{0},
$$

i.e., $F(\lambda, u, \theta)=0$.

Now, let us prove that $u_{k} \rightarrow u$ strongly in $H_{0}^{1}\left(\Omega_{0}\right) \cap H^{2}\left(\Omega_{0}\right)$. By (3.5) and (3.6) we deduce

$$
\begin{aligned}
& \int_{\Omega_{0}}\left\langle A \nabla\left(u_{k}-u\right), \nabla\left(u_{k}-u\right)\right\rangle \\
= & \int_{\Omega_{0}}\left\langle A \nabla u_{k}, \nabla u_{k}\right\rangle+\int_{\Omega_{0}}\langle A \nabla u, \nabla u\rangle-2 \int_{\Omega_{0}}\left\langle A \nabla u, \nabla u_{k}\right\rangle \\
= & \int_{\Omega_{0}}\left\langle\left(A-A_{k}\right) \nabla u_{k}, \nabla u_{k}\right\rangle+\int_{\Omega_{0}}\left(-f_{k} u_{k}-f u+2 f u_{k}\right) \\
= & o(1),
\end{aligned}
$$


because $A_{k} \rightarrow A$ in $C^{0}\left(\Omega_{0}\right)$ and $u_{k} \rightarrow u$ strongly in $L^{\frac{2 n}{n-2}}\left(\Omega_{0}\right)$. Moreover, we also have

$$
\begin{aligned}
& \int_{\Omega_{0}}\left(\operatorname{div}\left(A \nabla\left(u_{k}-u\right)\right)\right)^{2} \\
= & \int_{\Omega_{0}}\left(\operatorname{div}\left(\left(A-A_{k}\right) \nabla u_{k}\right)-f_{k}+f\right)^{2} \\
\leq & 2 \int_{\Omega_{0}}\left(\operatorname{div}\left(\left(A-A_{k}\right) \nabla u_{k}\right)\right)^{2}+2 \int_{\Omega_{0}}\left(f_{k}-f\right)^{2} \\
= & o(1),
\end{aligned}
$$

because $A_{k} \rightarrow A$ in $C^{1}\left(\Omega_{0}\right)$ and $u_{k} \rightarrow u$ strongly in $L^{\frac{2(n+2)}{n-2}}\left(\Omega_{0}\right)$. Then the claim follows directly from Remark 3.1.

Let us check assumption ii) of Theorem 3.2.

Proposition 3.1. $(0,0)$ is a regular value of $F$.

Proof. Let $\left(\lambda_{0}, u_{0}, \theta_{0}\right) \in U \times V$ such that $F\left(\lambda_{0}, u_{0}, \theta_{0}\right)=(0,0)$. We shall prove that if $(\lambda, u) \in X$ is such that

$$
\left\{\begin{array}{l}
F^{\prime}\left(\lambda_{0}, u_{0}, \theta_{0}\right)[\lambda, u]=0 \\
\left\langle F^{\prime}\left(\lambda_{0}, u_{0}, \theta_{0}\right)[\theta],(\lambda, u)\right\rangle_{Z}=0 \quad \text { for any } \theta \in Y
\end{array} \Rightarrow \lambda=0 \quad \text { and } \quad u \equiv 0\right.
$$

Without loss of generality we can assume $\theta_{0}=0$. Then $\Theta_{0}=I$ and by (3.2a) and (3.2b) condition $F\left(\lambda_{0}, u_{0}, \theta_{0}\right)=(0,0)$ reads as

$$
\left\{\begin{array}{l}
\int_{\Omega_{0}}\left(\left|\nabla u_{0}\right|^{2}-\left|u_{0}\right|^{p+1}-\lambda_{0} u_{0}^{2}\right) d x=0, \\
\Delta u_{0}+\left|u_{0}\right|^{p-1} u_{0}+\lambda_{0} u_{0}=0 \quad \text { in } \Omega_{0}, \quad u=0 \quad \text { on } \partial \Omega_{0} .
\end{array}\right.
$$

Moreover by (3.3) and (3.4) condition (3.7) can be rephrased as

$$
\left\{\begin{array}{l}
\int_{\Omega_{0}}\left\{2 \nabla u_{0} \nabla u-\left((p+1)\left|u_{0}\right|^{p-1} u_{0}+2 \lambda_{0} u_{0}\right) u-\lambda u_{0}^{2}\right\} d x=0 \\
\Delta u+\left(p\left|u_{0}\right|^{p-1}+\lambda_{0}\right) u+\lambda u_{0}=0 \quad \text { in } \Omega_{0}, \quad u=0 \quad \text { on } \partial \Omega_{0}
\end{array}\right.
$$

and

$$
\begin{aligned}
& \lambda \int_{\Omega_{0}}\left\{\nabla u_{0} \cdot\left[(\operatorname{div} \theta) \nabla u_{0}-\left(\theta^{\prime}+{ }^{t} \theta^{\prime}\right) \nabla u_{0}\right]-\left(\left|u_{0}\right|^{p+1}+\lambda_{0} u_{0}^{2}\right)(\operatorname{div} \theta)\right\} d x \\
& \quad+\int_{\Omega_{0}}\left\{\operatorname{div}\left[(\operatorname{div} \theta) \nabla u_{0}-\left(\theta^{\prime}+{ }^{t} \theta^{\prime}\right) \nabla u_{0}\right]+\left(\left|u_{0}\right|^{p-1} u_{0}+\lambda_{0} u_{0}\right)(\operatorname{div} \theta)\right\} u d x \\
& =0, \quad \forall \theta \in Y .
\end{aligned}
$$


We can simplify expression (3.10). Indeed, taking into account that

$$
\Delta u_{0}+\underbrace{\left|u_{0}\right|^{p-1} u_{0}+\lambda_{0} u_{0}}_{=g\left(u_{0}\right)}=0 \quad \text { in } \Omega_{0}, \quad u=0 \quad \text { on } \partial \Omega_{0},
$$

we have

$$
\begin{aligned}
& \operatorname{div}\left[(\operatorname{div} \theta) \nabla u_{0}-\left(\theta^{\prime}+{ }^{t} \theta^{\prime}\right) \nabla u_{0}\right] \\
= & \operatorname{div}\left(\theta \Delta u_{0}\right)-\Delta\left(\theta \nabla u_{0}\right) \\
= & -\operatorname{div}\left(g\left(u_{0}\right) \theta\right)-\Delta\left(\theta \nabla u_{0}\right) \\
= & -g\left(u_{0}\right)(\operatorname{div} \theta)-g^{\prime}\left(u_{0}\right) \nabla u_{0} \theta-\Delta\left(\theta \nabla u_{0}\right) .
\end{aligned}
$$

Moreover,

$$
\int_{\Omega_{0}} \Delta\left(\theta \nabla u_{0}\right) u d x=-\int_{\partial \Omega_{0}} \theta \nabla u_{0} \partial_{v} u d x+\int_{\Omega_{0}} \theta \nabla u_{0} \Delta u d x
$$

Therefore, (3.10) reads as

$$
\begin{aligned}
0= & \lambda \int_{\Omega_{0}}\{[g\left(u_{0}\right) u_{0}(\operatorname{div} \theta)+g^{\prime}\left(u_{0}\right) u_{0} \nabla u_{0} \theta+\theta \nabla u_{0} \underbrace{\Delta u_{0}}_{=-g\left(u_{0}\right)}]-\underbrace{\left(\left|u_{0}\right|^{p+1}+\lambda_{0} u_{0}^{2}\right)}_{=g\left(u_{0}\right) u_{0}}(\operatorname{div} \theta)\} d x \\
& -\lambda \int_{\partial \Omega_{0}} \theta \nabla u_{0} \partial_{v} u_{0} d x+\int_{\Omega_{0}}\left\{[-g\left(u_{0}\right) u(\operatorname{div} \theta)-g^{\prime}\left(u_{0}\right) u \nabla u_{0} \theta-\theta \nabla u_{0} \underbrace{\Delta u}_{=-g^{\prime}\left(u_{0}\right) u-\lambda u_{0}}]\right. \\
& +\underbrace{\left(\left|u_{0}\right|^{p-1} u_{0}+\lambda_{0} u_{0}\right)}_{=g\left(u_{0}\right)}(\operatorname{div} \theta) u\} d x+\int_{\partial \Omega_{0}} \theta \nabla u_{0} \partial_{\nu} u d x \\
= & \lambda \int_{\Omega_{0}} \underbrace{\left(g^{\prime}\left(u_{0}\right) u_{0}-g\left(u_{0}\right)+u_{0}\right)}_{=(p-1)\left|u_{0}\right|^{p-1} u_{0}+u_{0}} \theta \nabla u_{0} d x+\int_{\partial \Omega_{0}} \theta \nabla u_{0}\left(\partial_{\nu} u-\lambda \partial_{\nu} u_{0}\right) d x .
\end{aligned}
$$

Now, we prove that $\lambda=0$. Indeed by taking deformations $\theta$ which take fix the boundary of $\Omega_{0}$ by (3.12) we get

$$
\lambda \int_{\Omega_{0}}\left[(p-1)\left|u_{0}\right|^{p-1} u_{0}+u_{0}\right] \theta \nabla u_{0} d x=0 \quad \text { for any } \theta \in V, \quad \theta=0 \quad \text { on } \partial \Omega_{0} .
$$

If $\lambda \neq 0$ then we necessarily have

$$
u_{0}\left[(p-1)\left|u_{0}\right|^{p-1}+1\right] \nabla u_{0}=0 \quad \text { a.e. in } \Omega_{0},
$$

and so $u_{0} \nabla u_{0}=0$ a.e. in $\Omega$. This is not possible because $u_{0}$ solves (3.11) and by the unique continuation theorem in [2] we know that meas $\left\{x \in \Omega_{0}: u_{0}(x)=0\right\}=\operatorname{meas}\left\{x \in \Omega_{0}\right.$ : $\left.\nabla u_{0}(x)=0\right\}=0$. 
Since $\lambda=0$ by (3.12) we deduce that

$$
\int_{\partial \Omega_{0}} \theta \nabla u_{0} \partial_{\nu} u d x=0 \text { for any } \theta \in Y
$$

and arguing exactly as in [26, pp. 313-314], we deduce that $u=0$. That concludes the proof.

Proposition 3.2. For any $\theta \in \Xi$ as in (3.1) there exists $\lambda_{\theta} \in\left(0, \lambda_{1}\left(\Omega_{\theta}\right)\right)$ such that

$$
\lambda_{\theta}=2 \max _{\Omega_{\theta}} u_{\lambda_{\theta}} .
$$

Proof. Let $\theta \in \Xi$ as in (3.1) and let us consider the perturbed domain $\Omega_{\theta}$. For any $\lambda \in\left(0, \lambda_{1}\left(\Omega_{\theta}\right)\right)$ let $u_{\lambda}$ be the least energy positive solution on the domain $\Omega_{\theta}$, which is non-degenerate because of Theorem 3.1. Therefore, by the Implicit function Theorem we deduce that the map $\lambda \rightarrow u_{\lambda}$ is continuous. Let us consider the continuous function

$$
f(\lambda):=\lambda-2\left\|u_{\lambda}\right\|_{L^{\infty}\left(\Omega_{\theta}\right)}, \quad \lambda \in\left(0, \lambda_{1}\left(\Omega_{\theta}\right)\right) .
$$

Since

$$
\lim _{\lambda \rightarrow 0}\left\|u_{\lambda}\right\|_{L^{\infty}\left(\Omega_{\theta}\right)}=+\infty \quad \text { and } \quad \lim _{\lambda \rightarrow \lambda_{1}\left(\Omega_{\theta}\right)}\left\|u_{\lambda}\right\|_{L^{\infty}\left(\Omega_{\theta}\right)}=0,
$$

(see [14] and the classical bifurcation theory, respectively), there exists $\lambda_{\theta}$ such that $f\left(\lambda_{\theta}\right)=0$ and the claim (3.13) follows.

Proof of Theorem 1.2. It follows immediately by Theorem 3.1 and Proposition 3.2.

\section{Acknowledgements}

A. Pistoia was partially supported by Fondi di Ateneo "Sapienza" Università di Roma (Italy). G. Vaira was partially supported by PRIN 2017JPCAPN003 "Qualitative and quantitative aspects of nonlinear PDEs".

\section{References}

[1] A. L. Amadori, F. Gladiali, M. Grossi, A. Pistoia, and G. Vaira, A complete scenario on nodal radial solutions to the brézis-nirenberg problem in low dimensions, https://arxiv.org/pdf/2010.12311.pdf.

[2] N. Aronszajn, A. Krzywicki, and J. Szarski, A unique continuation theorem for exterior differential forms on Riemannian manifolds, Ark. Mat., 4 (1962), 417-453.

[3] F. V. Atkinson, H. Brezis, and L. A. Peletier, Nodal solutions of elliptic equations with critical Sobolev exponents, J. Differential Equations, 85(1) (1990), 151-170.

[4] T. Aubin, Espaces de Sobolev sur les variétés riemanniennes, Bull. Sci. Math., 100(2) (1976), 149-173. 
[5] G. Bianchi and H. Egnell, A note on the Sobolev inequality, J. Funct. Anal., 100(1) (1991), 18-24.

[6] H. Brezis and L. Nirenberg, Positive solutions of nonlinear elliptic equations involving critical sobolev exponents, Commun. Pure Appl. Math., 36(4) (1983), 437-477.

[7] L. A. Caffarelli, B. Gidas, and J. Spruck, Asymptotic symmetry and local behavior of semilinear elliptic equations with critical Sobolev growth, Commun. Pure Appl. Math., 42(3) (1989), 271-297.

[8] A. Capozzi, D. Fortunato, and G. Palmieri, An existence result for nonlinear elliptic problems involving critical Sobolev exponent, Ann. Inst. H. Poincaré Anal. Non Linéaire, 2(6) (1985), 463-470.

[9] G. Cerami, S. Solimini, and M. Struwe, Some existence results for superlinear elliptic boundary value problems involving critical exponents, J. Funct. Anal., 69(3) (1986), 289-306.

[10] Y. Dammak, A non-existence result for low energy sign-changing solutions of the BrezisNirenberg problem in dimensions 4, 5 and 6, J. Differential Equations, 263(11) (2017), 75597600 .

[11] M. del Pino, J. Dolbeault, and M. Musso, The Brezis-Nirenberg problem near criticality in dimension 3, J. Math. Pures Appl., 83(12) (2004), 1405-1456.

[12] O. Druet, Elliptic equations with critical Sobolev exponents in dimension 3, Ann. Inst. H. Poincaré Anal. Non Linéaire, 19(2) (2002), 125-142.

[13] P. Esposito, A. Pistoia, and J. Vétois, The effect of linear perturbations on the Yamabe problem, Math. Ann., 358(1-2) (2014), 511-560.

[14] Z. C. Han, Asymptotic approach to singular solutions for nonlinear elliptic equations involving critical sobolev exponent, Annales de l'I.H.P. Analyse non linéaire, 8(2) (1991), 159-174.

[15] A. Iacopetti and F. Pacella, A nonexistence result for sign-changing solutions of the BrezisNirenberg problem in low dimensions, J. Differential Equations, 258(12) (2015), 4180-4208.

[16] A. Iacopetti and G. Vaira, Sign-changing tower of bubbles for the Brezis-Nirenberg problem, Commun. Contemp. Math., 18(1) (2016), 1550036.

[17] A. Iacopetti and G. Vaira, Sign-changing blowing-up solutions for the Brezis-Nirenberg problem in dimensions four and five, Ann. Sc. Norm. Super. Pisa Cl. Sci., 18(1) (2018), 138.

[18] A. M. Micheletti, A. Pistoia, and J. Vétois, Blow-up solutions for asymptotically critical elliptic equations on Riemannian manifolds, Indiana Univ. Math. J., 58(4) (2009), 1719-1746.

[19] M. Musso and A. Pistoia, Multispike solutions for a nonlinear elliptic problem involving the critical Sobolev exponent, Indiana Univ. Math. J., 51(3) (2002), 541-579.

[20] M. Musso and D. Salazar, Multispike solutions for the Brezis-Nirenberg problem in dimension three, J. Differential Equations, 264(11) (2018), 6663-6709.

[21] A. Pistoia, A generic property of the resonance set of an elliptic operator with respect to the domain, Proc. Roy. Soc. Edinburgh Sect. A, 127(6) (1997), 1301-1310.

[22] B. Premoselli, Towers of bubbles for yamabe-type equations and for the brézis-nirenberg problem in dimensions $n \geq 7$, https://arxiv.org/abs/2009.01515.

[23] F. Quinn, Transversal approximation on Banach manifolds, in Global Analysis (Proc. Sympos. Pure Math., Vol. XV, Berkeley, Calif., 1968), pages 213-222. Amer. Math. Soc., Providence, R.I., 1970.

[24] O. Rey, The role of the Green's function in a nonlinear elliptic equation involving the critical Sobolev exponent, J. Funct. Anal., 89(1) (1990), 1-52.

[25] F. Robert and J. Vétois, Sign-changing blow-up for scalar curvature type equations, Commun. Partial Differential Equations, 38(8) (2013), 1437-1465. 
[26] J.-C. Saut and R. Temam, Generic properties of nonlinear boundary value problems, Commun. Partial Differential Equations, 4(3) (1979), 293-319.

[27] P. N. Srikanth, Uniqueness of solutions of nonlinear dirichlet problems, Differential Integral Equations, 6(3) (1993), 663-670.

[28] G. Talenti, Best constant in Sobolev inequality, Ann. Mat. Pura Appl., 110 (1976), 353-372.

[29] K. Uhlenbeck, Generic properties of eigenfunctions, Amer. J. Math., 98(4) (1976), 1059-1078.

[30] G. Vaira, A new kind of blowing-up solutions for the Brezis-Nirenberg problem, Calc. Var. Partial Differential Equations, 52(1-2) (2015), 389-422. 